\title{
Assessing the effect of weather on human outdoor perception using Twitter
}

\author{
Laura Giuffrida
}

Thesis supervised by

Professor Otto Klemm

Professor Edzer Pebesma

Professor Joaquin Torres-Sospedra 




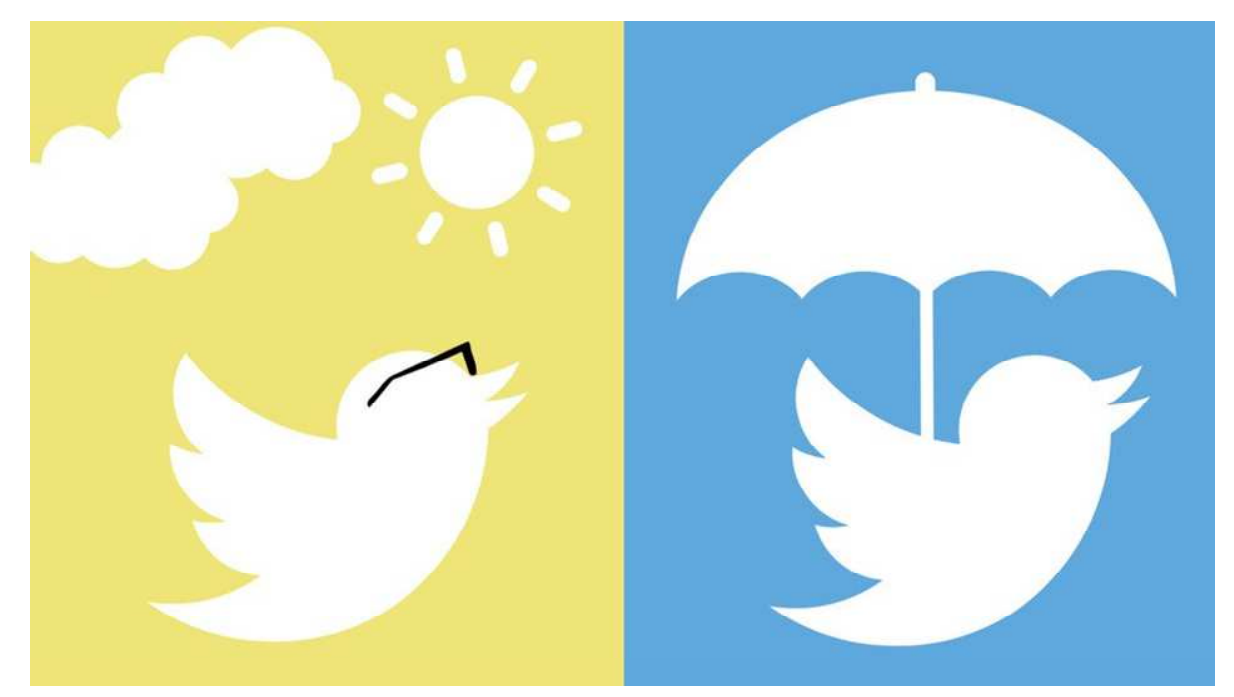

\title{
Assessing the effect of weather on human outdoor perception using Twitter
}

\author{
Laura Giuffrida
}

Thesis supervised by

Professor Otto Klemm

Professor Edzer Pebesma

Professor Joaquin Torres-Sospedra

Master of Science in Geospatial Technologies | February 20, 2017

Ifgi 



\section{Contents}

\section{Abstract}

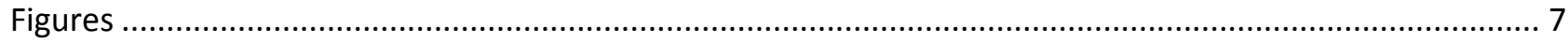

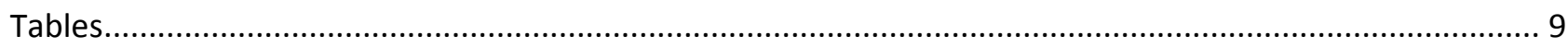

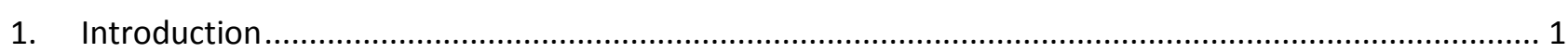

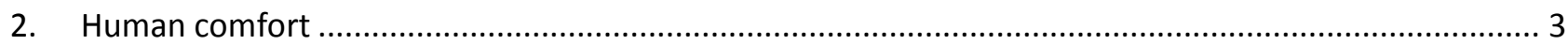

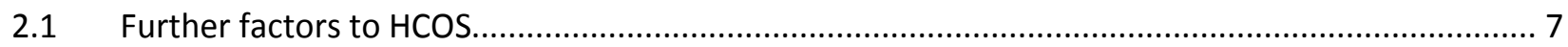

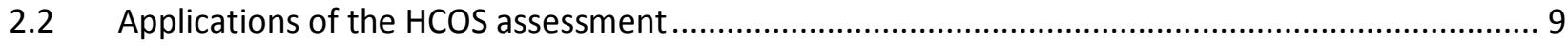

3. Twitter as a data source to understand and predict real-world phenomena ....................................... 11

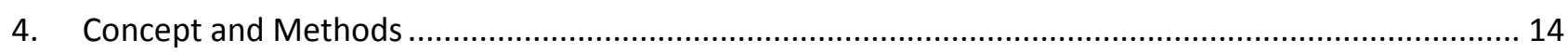

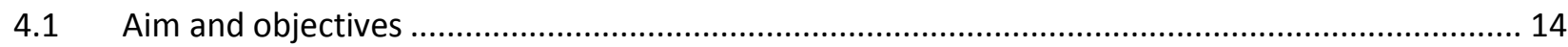

4.2 Study area

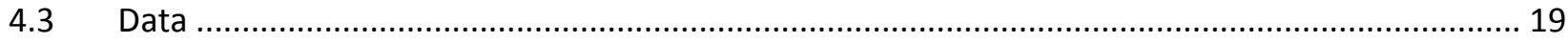

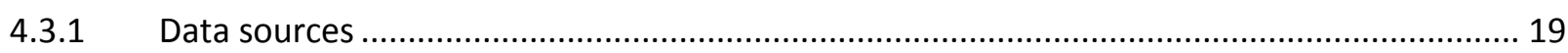

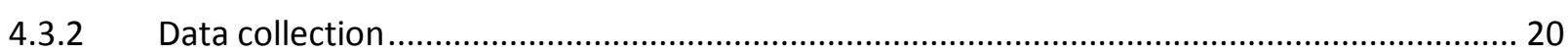

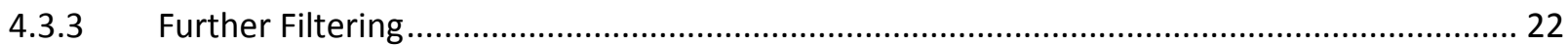

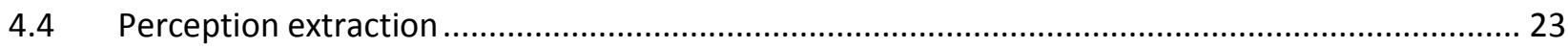

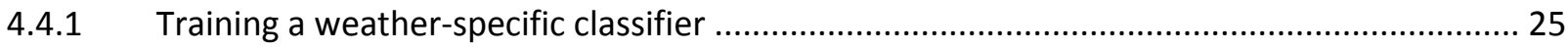

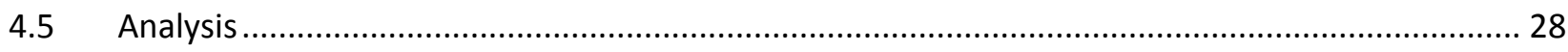

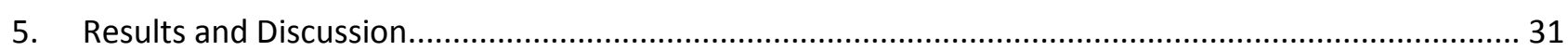

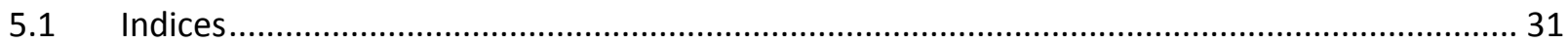

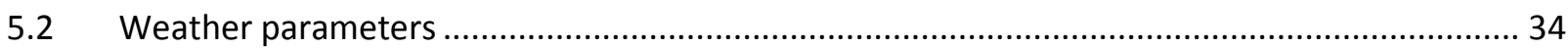

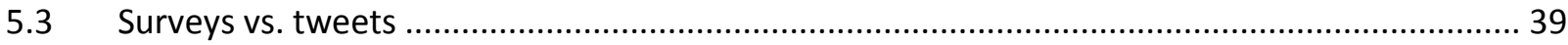

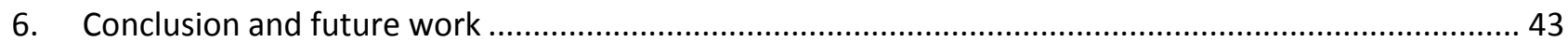

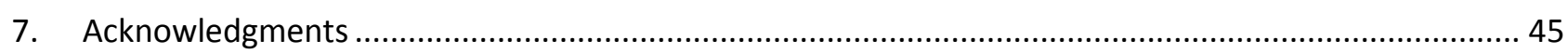

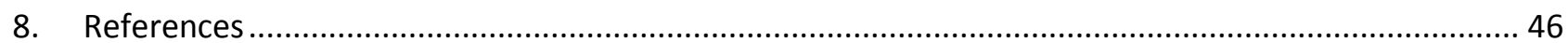




\section{Figures}

Figure 1. Top 15 most popular social networks worldwide by May 2016. Source: www.dreamgrow.com ... 11

Figure 2. Concept and methods scheme 14

Figure 3. Number of active Twitter users in millions by May 2016. Source: Worldwide Statista market analytics (Greenwood et al., 2016)..

Figure 4. Number of collected tweets by state. August 2016. Source. Twitter.

Figure 5. Climatic regions in USA. Source: www.ncdc.noaa.gov 16

Figure 6. Wind speed during October and November 1971 -2000. Source: www.nrcc.cornell.edu 16

Figure 7. Average temperature departure. Autumn 2012, 2013, 2014, 2015, 2016. Source:

www.nrcc.cornell.edu

Figure 8. Tweet on Indian Summer. Source: https://twitter.com.

Figure 9. REST API model vs. Streaming API model. Source: https://dev.twitter.com 19

Figure 10. Open Weather Map weather stations in New York city. Source: https://openweathermap.org.. 20

Figure 11. Data collection scheme 20

Figure 12. Raspberry Pi. Source: www.raspberrypi.org/about

Figure 13. Further filtering scheme

Figure 14. Tweet example of weather reports. Source: https://twitter.com

Figure 15. Tweet example of the use of slang words. Source: https://twitter.com 24

Figure 16. Tweet example of the use of emoticons. Source: https://twitter.com 24

Figure 17.Neutral tweet example. Source: https://twitter.com 25

Figure 18. Using the Natural Language Classifier service. Source: www.ibm.com/watson. 25

Figure 19. Average temperature comparison between October 2015 -2016. Source: www.nrcc.cornell.edu

Figure 20. USA and Japan according to Köppen classification by Ali Zafan. Source: Wikimedia Commons ... 29

Figure 21. Climate diagrams comparison. Aomori, New York, Pittsburg and Boston. Source:

www.klimadiagramme.de 30

Figure 22. Effective Temperature variation from Twitter data 31

Figure 23. Effective Temperature ranges applied to the Twitter data. 
Figure 24. Effective temperature thresholds at different months. Source: BARANOWSKA and GABRYL, 1981.

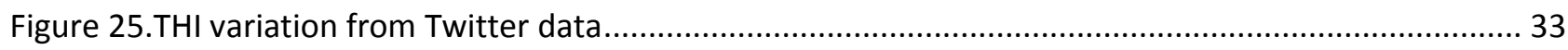

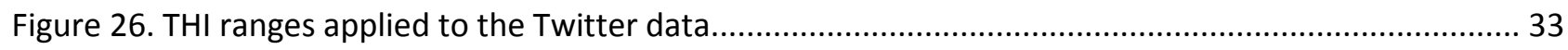

Figure 27. Variation of comfort sensation with air temperature. Source: SASAKI ET AL. (2000) .................. 34

Figure 28. Variation of comfort sensation with Temperature from Twitter data......................................... 34

Figure 29. Variation of comfort sensation with Humidity. Source: SASAKI ET AL. (2000) ............................ 35

Figure 30. Variation of comfort sensation with Humidity from Twitter data ............................................ 35

Figure 31. Variation of comfort sensation with wind. Source: SASAKI ET AL. (2000) .................................. 36

Figure 32. Variation of comfort sensation with wind from Twitter data .................................................. 36

Figure 33. Variation of comfort sensation with weather description. Source: SASAKI ET AL. (2000)............. 36

Figure 34. Variation of comfort sensation with weather description from Twitter data ............................ 37

Figure 35. Variation of comfort sensation with cloud coverage from Twitter data...................................... 37

Figure 36. Total number of tweets for all weather parameters (a. Temperature, b. Humidity, c. Wind speed,

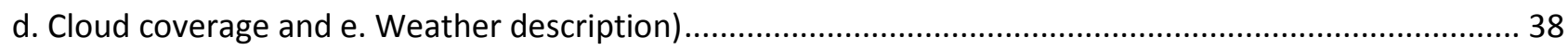

Figure 37. Example of the opinions reflected by tweets. Source: https://twitter.com .............................. 41

Figure 38. Tweet example of weather preferences in compliance with personal activities. Source: https://twitter.com 


\section{Tables}

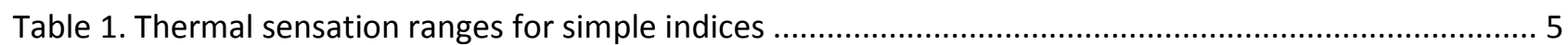

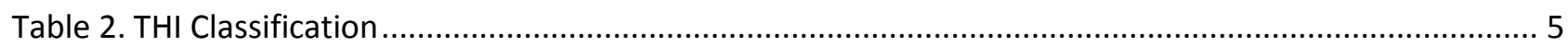

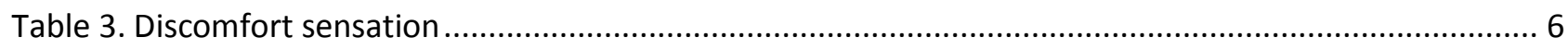

Table 4.Effective Temperature ranges and assessment scale............................................................... 31

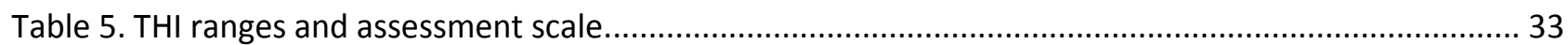




\begin{abstract}
Human Comfort in Outdoor Spaces (HCOS) is linked to physical, physiological and psychological responses of people to environmental variables. Previous studies have established comfort ranges for these variables through questionnaires, reaching only small populations. However, larger amounts of data could not only generate more robust results in local studies, but also allow the possibility of creating an approach that could be applied into a wider range of weather conditions and different climates. This thesis describes a new methodology to assess people's perception of weather based on human responses to weather conditions extracted from tweets, with the purpose of establishing comfort ranges for environmental variables. Tweets containing weather-associated keywords were collected using the Twitter API and then linked to real-time meteorological data acquired from the Open Weather Map API, which provides weather variables measured nearby the locations in which the tweets were posted. Afterwards, people's perception of weather was extracted from the tweets using a classifier trained specifically on weather data that identified irrelevant, neutral, positive and negative tweets. The obtained tweets and their related meteorological data were analyzed to establish comfort ranges. Comparing the resulting ranges to others obtained in previous studies, a generally good agreement was found with the indices Effective Temperature (ET) and Termohygrometric index (THI) derived from questionnaires, but the peak of comfort is shifted towards lower and higher temperatures, respectively. Regarding the single weather variables, the obtained comfort ranges are alike the ones found in previous research, in particular, the temperature comfort range matches perfectly at $20^{\circ} \mathrm{C}$ $-22^{\circ} \mathrm{C}$. Therefore, it was concluded that tweets can be used for the assessment of HCOS; not only the results of this methodology are comparable to the ones obtained in previous studies, but also the procedure itself shows new features and opportunities for future applications.
\end{abstract}





\section{Introduction}

In the past years, there has been an enormous growth in the use of microblogging platforms like Twitter. Millions of messages are posted daily, on real-time, covering a wide range of topics, including opinions and feelings concerning Twitter users' lives (Pak and Paroubek, 2010). Therefore, this platform has become a rich source of data for opinion mining and sentiment analysis, which aim at determining the attitude of a writer with respect to various topics (Kumari et al., 2015). Besides expressing views and opinions, tweets provide novel answers to classic questions and reports on current events. For this reasons, they could be used to predict, monitor, and cope with many different types of events, from simple matters of daily life to massive crises in the global scale.

Moreover, Twitter is the container of various types of available user-generated data that was published using smartphones and other GPS-enabled devices. Under this perspective, tweets are geographic information that can support decision making processes, when combined with other kinds of information such as sensors installed in certain locations with specific scopes. In this context, tweets highlight the potential of using citizen as sensors to improve people's quality of life, which is a concept that is recurrently used by authors like Goodchild and Sheth. They and many others see citizens as a network of human sensors with billions of components that share their observations and views using mobile devices and Web services. They use their background knowledge, experience and perception to synthesize and interpret local information (Crowley et al., 2013).

It is also true that most tweets contain little information value and sometimes their reports do not correspond to the actual location of the user. Nevertheless, it is the aggregation of them that can generate meaningful content that can be translated into knowledge (Paul and Dredze, 2011). Twitter provides information that is not available in other intelligent sources, since it includes huge amounts of available data generated from users located close to the action (Panasyuk et al., 2013).

Several studies have demonstrated that the aggregation of tweets can provide valuable insights into a population (Paul and Dredze, 2011). Interesting patterns of aggregate sentiments, opinions and reports have been identified and used to measure and predict real-world events (Hannak et al., 2012). However, the nature of Twitter data requires practical approaches to obtain meaningful contents from it.

At analytical level, there are several technological innovations that help making sense of the large number of tweets (Kumari et al., 2015), so they can be efficiently used for social studies (Pak and Paroubek, 2010). For example, machine-learning methods have been trained on annotations to extract emotions from this kind of data, giving insights into users' feelings towards specific topics and enabling emotion-temporal analysis Roberts et al., 2012).

In this context, this project aims to assess people's perception on weather based on human responses to weather conditions extracted from tweets to establish Human Comfort ranges that could be used in several fields such as biometeorology and urban planning.

So far, these ranges have been established through interviews and questionnaires, reaching only small populations. However, larger amounts of data could not only generate more robust results in local studies, but also allow the possibility of creating an approach that can be applied into a wider range of weather conditions and different climates. For these reasons, an alternative methodology for the assessment of Human Comfort should be proposed. 
Introduction

The remainder of this thesis is organized as follows. Chapter 2 introduces the concept of human comfort, as well as the evolution of its assessment and the importance and applications of comfort ranges. Chapter 3 presents practical examples of tweets used as data source for social research. Chapter 4 will illustrate the data collection process, the choice of the study area and the methodology implemented for the perception extraction. Chapter 5 presents the resulting comfort ranges and their comparison with other comfort ranges established in previous studies. It also shows the pros and cons of tweets and questionnaires, when analyzing human comfort. Finally, Chapter 6 summarizes the conclusions and motivates future work. 


\section{Human comfort}

In this chapter, the concept of human comfort will be introduced, as well as the evolution of its assessment in the last decades. Moreover, the importance and applications of comfort ranges will be illustrated.

Human comfort is a state of feeling, even if it is not associated to any specific sense organ. It is generally connected to pleasant conditions that are related to health and happiness. In specific, human thermal comfort has been defined as "the state of mind which expresses satisfaction with the thermal environment" (Gagge et al., 1967). This means that thermal comfort is linked to the psychological responses of people to environmental variables.

In the past decades, several scientists from different disciplines have tried to define thermal comfort scales for different reasons. At the beginning, they carried out experiments conducted in indoor spaces that allowed the variation of a single parameter while maintaining the rest of them constant. By applying this methodology, they discovered important features of the comfort perception.

For example, Gagge et al. (1967) performed experiments on unclothed subjects exposed to various temperatures. They observed that when body temperatures tended to the physiological neutrality people felt more comfortable, even if the same temperatures were related to discomfort in other circumstances.

Gagge et al. (1968) completed a second series of experiments, but this time they considered subjects doing physical activities. They concluded that warm discomfort and temperature perception are less affected by changes in temperature during exercise that during rest. They also described a comfortable zone for steady exercise, considering metabolism rates and perspiration, as well as humidity, wind speed and air temperatures. The lower boundary of this zone coincided with skin sweat equal to zero and the upper boundary corresponded to $65 \%$ wetness of the skin.

Fanger (1972) performed chamber experiments, measuring sweat rate and skin temperature of people who considered themselves comfortable at various metabolic rates. He concluded that the ideal conditions for thermal comfort can be deduced from the metabolic rate, clothing insulation and environmental conditions. His model, based on indoor climate, allowed the calculation of the Predicted Mean Vote (PMV), which was one of the most popular comfort equations during the 1990s.

Then, the scientific community shifted its interest to the Human Comfort in Outdoor Spaces (HCOS), motivated by the need to explore the real conditions in which humans perform their everyday activities. Thus, the PMV equation was extended by Jendritzky (1979) to be applied in outdoor conditions, using the KlimaMichel-Model. Nevertheless, PMV was very hard to use for people who did not belong to the thermophysiology field and, therefore, other indices were proposed.

Some of them still focused on human metabolism and heat balance equations (e.g. Psychological Subjective Temperature, Standard Effective temperature, Psychological Equivalent Temperature, Perceived Temperature), but others moved their emphasis into the monitoring of environmental variables. The latter indices were also called simple indices because they neither consider thermo-physiological parameters into their formula nor are they built on heat balance models. Instead, they only show the joint effects on the human body of several meteorological variables, based only on empirical research or some general theoretical considerations. Over time simple indices outmatched the complex ones due to their easier implementation and the fact that they do not require invasive measurements (e.g. rectal temperature) (Blazejczyk et al., 2012). 
The following is a selection of comfort indices based on meteorological measurements. Among them, Heat Index, Humidex, Effective Temperature, Wet-bulb globe temperature, Apparent Temperature and Wind Chill:

- Heat Index (HI) combines temperature and relative humidity to define an apparent temperature. It is applicable in cases in which the temperatures are above $20^{\circ} \mathrm{C}$. It assumes that when humidity is high, the evaporation of water is low, which means that the body would retain more heat than under dry air conditions.

- Humidex describes as how hot humid weather is perceived by an average person. It combines temperature and vapor pressure.

- Effective Temperature (ET) defines the thermal exchange between a human organism and its environment, considering wind speed, relative humidity and temperature. It assumes normal atmospheric pressure values and normal body temperature.

- Wet-bulb globe temperature (WBGT) is one of the most used heat stress indices in the world. It is a kind of apparent temperature used to assess the influence of temperature, humidity, wind speed, and sunlight on bodies.

- Apparent Temperate (AT) is a modification of the ambient temperature based on humidity levels. There are two formulas for AT. The first includes solar radiation, vapor pressure, wind speed and temperature, while the second one excludes solar radiation.

- Wind Chill (WC) expresses the cooling power of wind in total shade and without evaporation. It includes temperature and wind speed values.

The characterization of Human Comfort includes also the definition of verbal categories for comfort perception rages that accompany the indices. These categories have also been the focus of numerous studies in for years. Winslow et al. (1937) presented a way of classifying comfort sensation, using only 5 classes (very pleasant, pleasant, indifferent, unpleasant and very unpleasant), while Nevins et al. (1966) decided to classify thermal sensation using 7 classes (cold, slightly cold, cool, comfortable, slightly warm, warm and hot). Gagge et al. (1967) analyzed both comfort sensation and thermal sensation of unclothed subjects exposed at various temperatures, obtaining combined sensations as pleasantly warm or pleasantly cool. Later, Fanger (1972) adapted Nevins' classification to his PMV equation, but his output ranges were criticized later for being abstract numbers, instead of temperature values that could be easily be compared. Jendrizky et al. (1979) developed a human biometeorological classification for thermal stress on human bodies using PMV values. They proposed a six-classes table (no stress, resting range, slight stress, moderate stress, strong stress and very strong stress) to describe also outputs ranges consisting of abstract numbers.

The thermal sensation classification proposed by Nevins is currently the most used, and over time other classes were added. The following table shows the comfort perception ranges of the 5 indices presented above (Table 1), which provide temperature as the output value of their formula, accompanied by an extended version of Nevins' classification. 
Table 1. Thermal sensation ranges for simple indices

\begin{tabular}{|c|c|c|c|c|c|}
\hline HI & Hum & ET & WBGT & WC & Thermal sensation \\
\hline$>54$ & $>55$ & & $>30$ & & Sweltering \\
\hline $41-54$ & $45-55$ & $>27$ & $28-30$ & & Very hot \\
\hline $32-41$ & $40-45$ & $23-27$ & $24-28$ & & Hot \\
\hline $27-32$ & $30-40$ & $21-23$ & $18-24$ & & Warm \\
\hline & $<30$ & $17-21$ & $<18$ & $>-10$ & Comfortable \\
\hline & & $9-17$ & & $-27--10$ & Cool \\
\hline & & $1-9$ & & $-39--28$ & Cold \\
\hline & & $<1$ & & $-54--40$ & Very cold \\
\hline & & & & $<-55$ & Frosty \\
\hline & & & & & \\
\hline
\end{tabular}

In the past years, the European Union has been developing the Universal Thermal Climate Index (UTCI). It is based on recent scientific progress in human response related thermo-physiological modelling, therefore, is very sensitive to changes in wind speed, temperature, solar radiation and humidity. So far, UTCl is the most versatile index of HCOS and it is expected to become an international standard (Nass, 2017). Several research efforts have been made to evaluate UTCl outputs. Blazejczyk et al. (2012), for instance, presented a comparison analysis of $\mathrm{UTCl}$ and some of the most used thermal indices. He concluded that all the indices based on heat balance models had a higher correlation with UTCI than the simple ones, mainly because they do not consider solar radiation in their formula. The only exception was the Effective Temperature, which showed the second highest correlation (96.7), after Standard Effective temperature (97.5). ET can also be applied under a wider range of thermal conditions, in comparison to other simple indices, as we can see in table 1.

Nevertheless, ET can be also estimated through the application of Thom's discomfort index, which is also called Thermohygrometric Index (THI). It was originally used to calculate discomfort due to heat stress, but over time, it has been applied into a broader variety of conditions. An assessment scale has also been established for THI. In this, comfort conditions occur between $15{ }^{\circ} \mathrm{C}$ and $20{ }^{\circ} \mathrm{C}$. Under $15{ }^{\circ} \mathrm{C}$, thermogenic mechanisms are required to fight cold stress and, over $20^{\circ} \mathrm{C}$, the perspiration system must be activated to prevent overheating. As one may see in Table 2, the THI assessment scale is less detailed when heat increases. Therefore, further research has introduced a classification table for summer (Table 3), based on the discomfort sensation. Nowadays, THI is widely used to estimate the differences of urban and rural bioclimatological conditions (Unger, 1999).

Table 2. THI Classification

\begin{tabular}{|c|c|}
\hline THI & Thermal sensation \\
\hline$>30$ & Torrid \\
\hline $26.4-29.9$ & Very hot \\
\hline $20-26.5$ & Hot \\
\hline $15-20$ & Comfortable \\
\hline $13-15$ & Cool \\
\hline $1.7-13$ & Cold \\
\hline$-10-1.7$ & Very cold \\
\hline$-10--20$ & Extremely cold \\
\hline$-20-40$ & Glacial \\
\hline$<-40$ & Hyper glacial \\
\hline
\end{tabular}


Table 3. Discomfort sensation

\begin{tabular}{|c|c|}
\hline THI & Discomfort sensation \\
\hline$>21$ & Under $50 \%$ population feels discomfort \\
\hline $24-27$ & Over $50 \%$ population feels discomfort \\
\hline $27-29$ & Most of population feels discomfort \\
\hline $29-32$ & Everyone feels severe stress \\
\hline$>32$ & State of medical emergency \\
\hline
\end{tabular}

The application of indices has been proved to be very valuable to the assessment of HCOS and the definition of its comfort ranges. Nevertheless, it is not the only way. Several researches have studied the effects of single weather parameters on people's perception. Stathopoulos et al. (2004), for example, chose the parameters wind speed, air temperature, relative humidity and solar radiation. They investigated the relationship between the comfort level of human activities and these four major weather parameters in Montreal and exposed their effect on comfort perception and preferences. Similarly, Nikolopoulou and Lykoudis (2006) studied the same four weather parameters, but across 7 European cities and throughout a whole year, to inquire into the relationship between microclimatic and comfort conditions.

In other cases, the combination of wind speed, humidity, temperatures and solar radiation was not chosen. For instance, de Motingny et al. (2011) studied the effect of temperatures, precipitation intensity and sunlight hours on pedestrian fluctuations in 9 different cities located in the northern hemisphere and Thorsson et al. (2007) related the presence of people in open spaces to temperature, wind speed and sky clearness, excluding solar radiation due to the difficulties in measuring it. These three parameters were proven to influence people's perception of public open spaces as comfortable in different extends in Sweden and Japan. Sasaki et al. (2000) studied the relation between comfort sensation and meteorological factors in Northern Japan, by taking into account wind speed, relative humidity, temperatures and weather description. 


\subsection{Further factors to HCOS}

The study of weather parameters has revealed their strong influence on comfort perception. Nevertheless, it has been demonstrated that psychological factors may be as important as the physical ones (Nikolopoulou et al, 2003). Even if the complexity of the assessment of the Human Comfort in Outdoor Spaces (HCOS) has been discussed, there are still some factors that must be considered to define HCOS ranges.

First of all, adaptation, which implies all the actions that make humans better suited to survive a specific environment. It can be separated in three different categories physical, physiological and psychological.

Physical adaptation involves both personal changes such as modification of clothing or consumption of hot drinks and changes that people make to their surroundings to improve their comfort. Physiological adaptation implies a change in the functions of the human body because of repeated exposure to specific conditions. Finally, the psychological adaptation contemplates the fact that each person perceives the environment in a different way depending on the information that this person has about a certain situation ( $\mathrm{Ni}$ kolopoulou et al, 2003). This last category includes the effects of seasonal fluctuations and cultural differences on the comfort perception.

Seasonal fluctuations affect people's perception of weather because the average parameters for a certain season characterize the expectation of acclimatized residents; in other words, "expectations of seasonable weather conditions among residents may condition the physiological response to weather" (Stathopoulos et al., 2004). Consequently, the expectations on people's perception affect people's choice of clothing and, since people are prepared for a specific thermal sensation at that specific time, they may interpret any variation as dissatisfaction (Nikolopoulou and Lykoudis (2006). Nevertheless, the opposite can also be true, as Palutikof et al. (2004) discovered when studying people's responses to exceptional mild winters. They found that $69 \%$ of the interviewed believed that warm winters have a positive impact on their comfort, as well as in their leisure activities. Moreover, $66 \%$ of the people declared to prefer unusual warm weather to unusual cold weather and the idea of having more mild winters in the future was considered by them to be either pleasant or very pleasant.

Skewed results in comfort studies that compare two or more regions are usually related to cultural differences. For example, the study by Thorsson et al. (2007) on thermal comfort and outdoor activity exposed differences between human behavior related to sun and exposition time in South Japan and Sweden. They found out that thermal conditions have a smaller effect on the attendance to open spaces in Japan than in Sweden. They associated this result not only to climate, but also to cultural differences between the two countries. Since in Scandinavia the time spend outdoors is confined by cold weather and short sunshine hours and their ideal of beauty includes suntanned skin and, in Japan, the cold weather is rarely a problem and the ideal of beauty is light skin. Moreover, Palutikof et al. (2004) and his team, in another study, considered samples taken within Scotland and England to compare the results and attribute eventual differences in the results to cultural more than climatic differences. Also, de Motingny et al. (2011) studied the effects of temperatures, sunlight and precipitation on the number of pedestrians in nine different cities located across the northern hemisphere. He noticed that the pedestrian flow increased when it was not raining or snowing. But the degree of this variation changes across the different cities accordingly to the character of the local environment, the social aspects of walking and the inhabitants' adaptations to the local weather. 
Consequently, due to physical, physiological and psychological adaptation the perception of the same weather conditions and weather preferences may vary in relation to where people live. For example, $\mathrm{Ni}$ kolopoulou et al. (2006) carried out a study in four different open spaces in the city of Cambridge during spring, summer and winter. She and her team noticed that the greater number of people outside was found during the summer, so they concluded that people enjoyed feeling warm. However, considering their experience in other study areas, they argued that in warmer climates the situation would be the opposite and more people would be found outdoors when temperatures are lower than the average.

Furthermore, weather preferences may also be related to people's activities. People performing physical activities, like jogging or biking, prefer different weather conditions than people sitting in a square or walking to work. This is due to a variation in their metabolic rate and, consequently, in their thermal balance. However, it must be considered that activities may change the perception of thermal comfort as much as thermal comfort affects people's activities. For example, air temperatures have been proved to have a great impact on walking times and people's attendance to open spaces (de Motingny et al. 2011). 


\subsection{Applications of the HCOS assessment}

The health and comfort of people living in cities have been considered by the urban planning field through urban biometeorology. The field was established to evaluate the combined effect of weather, climate and air pollution on humans, by defining threshold values that can include not only measurements, but also the physiological aspect into the assessment (Mayer, 1993). Urban biometeorology is divided in two different aspects: the air quality and the thermal component of the urban climate, and it is very important to distinguish them. The thermal component, the one of our interest, is based on the idea of the perfect urban climate. It may be utopic, but can be approached by avoiding extreme conditions and minimizing climatic stress on citizens (Jendritzky et al., 1979). Studies suggest some general conditions in which people are bioclimatically comfortable: temperature between 21 and $27.5{ }^{\circ} \mathrm{C}$, relative humidity between 30 and $65 \%$, and wind speed up to $5 \mathrm{~m} / \mathrm{s}$ (Toy et al., 2007).

Urban biometeorology is also related to the assessment of the quality of urban living, which includes 9 factors: material wellbeing, health, political stability and security, family life, community life, climate, job security, political freedom and gender equality (Economist Intelligence Unit, 2005). Regarding the climate factor, the strategy they have been following is to rate cities highly when they remained close to $21^{\circ} \mathrm{C}$ all year around, and penalize places that have extremely cold or extremely hot temperatures. Yet, it was pointed out that some people enjoy hot weather and others like cold weather, and yet others enjoy seasonal changes. So, even if the climate factor principle rests on the assumption that mild climate is the most desirable one, which is a widespread presumption, it is often problematic in complex and heterogeneous societies (Diener et al., 1997).

Over time, the applications of the comfort perception analysis multiplied and its aim was not only to explore and quantify the impact of weather on human health, but also to understand how weather characteristics support specific social behaviors. In this context, architects and urbanists gained interest in the subject, since most of the activities that take place in cities are affected by weather parameters, which in turn influence the physical environment of the inhabitants. Having specific threshold values or comfort ranges for each of these weather parameters would be of great help at the early stages of urban design and planning. Knowing people's comfort requirements linked to everyday activities such as walking, biking and attending to public open spaces, urban planners can successfully create urban areas that attract large numbers of people and become economically profitable, modifying the image of a city (Nikolopoulou and Lykoudis, 2006).

Penwarden (1973), for example, established limits to wind speed that were acceptable or unacceptable for pedestrian areas. He used the Beaufort scale as a base for his studies. This table, which was built in 1805 to measure wind speeds, considers observations rather than accurate measurements, so Perwarden integrated it with calculations of wind force on the human body, its effects on balance and the effort needed to walk against it. The results show that speeds over $5 \mathrm{~m} / \mathrm{s}$ are at the beginning of the discomfort limit, those around $10 \mathrm{~m} / \mathrm{s}$ were classified as absolutely unpleasant and, finally, the speeds of about $20 \mathrm{~m} / \mathrm{s}$ are considered dangerous. The higher the wind speed the larger the consumption of energy to maintain walking speed and balance.

We can find an example of how comfort ranges provide useful information to urban planners in San Francisco, USA. There, the Recreation and Park Department has approved separated ordinances for sun and wind, based on comfort in outdoor spaces (Arens and Bosselmann, 1989). Since San Francisco presents a cool climate, the sun ordinance states that any decrease of sunlight is penalized. The wind ordinance, on the other 
hand, assumes that air temperatures are low and strong winds are translated into unpleasant cooling. Nevertheless, these criteria may be acceptable for the local climate, but in other climatic regions they will be incorrect and, consequently, they will lead to inappropriate decisions.

Other studies were concerned with the influence of weather on walking levels in urban areas. For instance, de Motingny et al. (2011) studied the effects of temperatures, sunlight and precipitation on the number of pedestrians in nine different cities located across the northern hemisphere. He noticed that the pedestrian flow increased when it was not raining or snowing. He also compared the urban features of the cities under study and concluded that air temperature, irradiation and precipitation effects can be controlled within some certain limits and, consequently, suggested microclimate manipulations through physical urban design to enhance walking.

It has been shown by Thorsson et al. (2007) that walking trips are more resistant to weather than public open space attendance, since the first is frequently necessary to complete routine paths or chores and the second one is mainly a voluntary activity. In fact, studies on this matter have demonstrated that the presence of people in open spaces is very sensitive to weather and any variation in this relation is related to cultural and climate factors. The sensibility of the attendance to some public open spaces can also be related to the nature of the space: its location and function in the city. Some squares are located nearby office areas are transportation nodes, while some others are located outside of the city center and are more dedicated to leisure activities. With this in mind, urban designers can consider the HCOS ranges to modify the characteristics of open spaces and their surroundings support stationary social activities. 


\section{Twitter as a data source to understand and predict real-world phenomena}

In this chapter, practical examples of tweets used as data source for social research will be presented to give a panoramic view of what has been done so far.

In the last years, social media has grown until the point in which it is almost ubiquitous. Thus, there is a proliferation of easily and quickly accessible data that could transform social science research. In this context, many researchers have started to collect data from social media platforms to document and monitor spontaneous expressions of moods, feelings, attitudes, opinions, activities and interactions (McCormick et al., 2015). What makes social media platforms an interesting data source is that they contain vast quantities of traces of human behavior related to a wide array of topics, in real-time and on large scale. While single messages contain little value, it is the aggregation of them that generates important insights into specific populations (Paul and Dredze, 2011).

Even though Facebook, YouTube and Instagram are the most popular social media platforms nowadays (Figure 1), Twitter seems to be the favorite for social research. This may be because Twitter makes it easier to find and follow conversations, which gives insights into people's interactions. Also, big events and news stories are easy to find as they are usually associated to a hashtag. Moreover, the Twitter API is more open and accessible than other social media platforms' APIs, which makes it more attractive to developers. This consequently increases the availability of tools like sentiment analysis and machine learning methods (which work well with Twitter data as tweets allow maximum 140 characters), as well as time series analysis. Finally, many researchers have a Twitter account and may feel more comfortable when using a familiar platform as a data source (Ahmed, 2015).

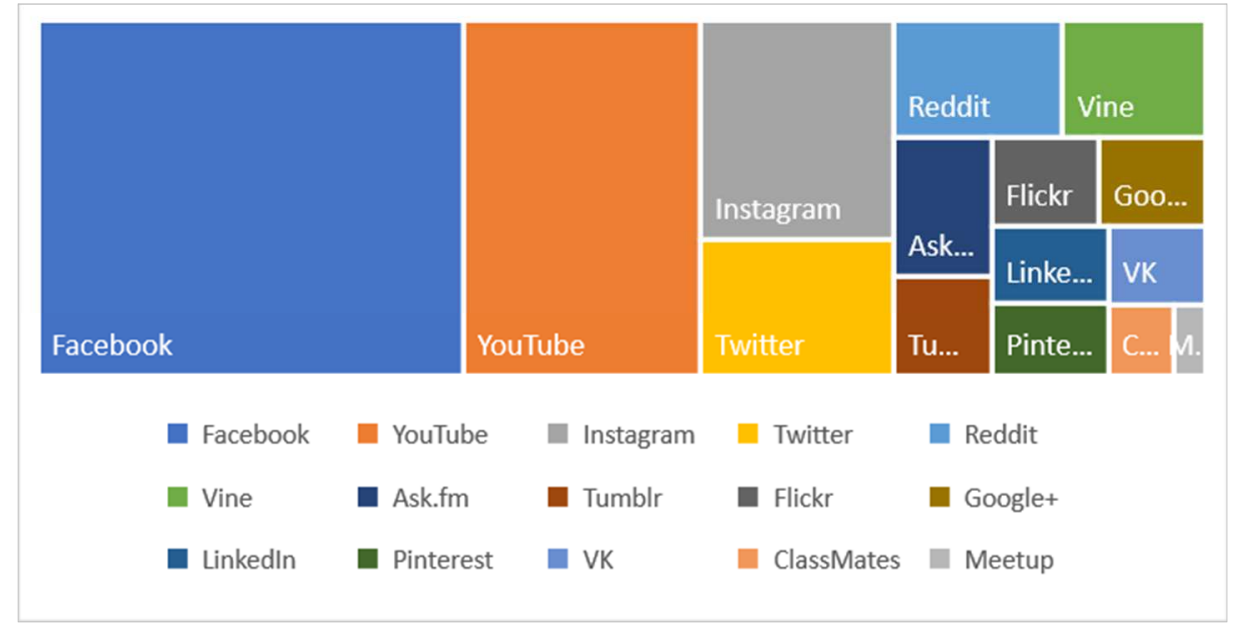

Figure 1. Top 15 most popular social networks worldwide by May 2016. Source: www.dreamgrow.com

In this context, Twitter data have proven to be useful in several different fields, for example, public health monitoring. Paul and Dredze (2011) collected more than one million tweets related to health disorders to track them over time and localize them geographically. They concluded that public health institutions and researchers should integrate the current methods to survey sicknesses and symptoms with Twitter data to increase both the range and the type of information that can be tracked. Krieck et al. (2011) argue that the detection of disease activity in an early stage, if followed by a fast response, can reduce the effect of epidemics. To address this challenge, they collected and analyzed twitter messages posted during sickness outbreaks and concluded that they can be relevant for public health threats detection. 
Twitter as a data source to understand and predict real-world phenomena

Tweets can also track political opinions or predict election results. Bekafigo and McBride (2013) investigated how citizens use Twitter to engage in politics. The results demonstrated that traditional political activist tweet about politics more often, but also some marginalized groups, such as racial minorities, participate in Twitter conversations, which may indicate that they have found a space to express their political opinions. Gerbaudo (2012) analyzed protest movements of the 21st century. He found a connection between the escalation of social media and the appearance of new kinds of protest. Tumasjan et al. (2010) collected tweets before the 2009 German election and concluded that the opinion trends reflected in their data paralleled the results of the election, while Scorik et al. (2012) studied the use of Twitter during elections in Singapore and found out that the accuracy was significantly lower than in the previous case, which suggests that media freedom, among other issues, might have led to inaccuracies in the estimations of the voting sentiment.

Cox and Pale (2011) studied how to use tweets to increase the accuracy of localized weather events reports and they concluded that the twitter stream does not contain enough contextual information for the spatiotemporal analysis they proposed and, therefore, the monitoring of localized weather events for many areas would not be possible. Nevertheless, they argued that it could be very useful during significant weather events like hurricanes or earthquakes. Lachan et al. (2014) collected tweets within \#nemo and \#bosnow (which were created to share information about the winter storm that affected USA and Canada in 2013) from the historical archives of Twitter. Their aim was to categorize different kinds of risk perception observed in national and local hashtags and found out that localized hashtags provide a stronger sense of relevance to people. Moreover, they suggested that since Twitter can allow government agencies to monitor public reactions in real-time, they should provide useful information through these kinds of hashtags.

Mood rhythms on large scales have been also observed through tweets, as it was demonstrated in a recent study carried out by a group of scientists from Cornell University. They measured and mapped the frequency of words expressing positive and negative emotions to show how individual mood varies from hour to hour, day to day, and across the seasons. They found that there are robust rhythms across diverse cultures but, in general, people tend to be in better mood during the morning (Asanet, 2012). Similarly, Biever (2010) used tweets to map moods on large scale, discovering the general emotional state of America over a period. But moods can be also modelled, as Bollen et al. (2011) demonstrated. They performed sentiment analysis on tweets, extracted six mood states and compared their results to a list of popular events. They found out that events in the social, political, cultural and economic domain have a significant and immediate effect on public mood.

Regardless of their application, the studies above and many others suggest that tweets are a good source to understand human activities and attitudes. They are spontaneously created and archived by humans, so they allow researchers to see what people are doing and saying, and how they feel about specific events while they are still happening. It is undeniable that they are an extraordinary form of data with huge research potential. Nevertheless, it must be appropriately managed and such a task presents several challenges (McCormick et al. 2016).

For example, even if Twitter's API offers a fast way to collect great amounts of data in real time, it provides a sample that only includes up to one percent of all tweets if a filter is not used, which implies a lack of transparency regarding how tweets are sampled within the stream (Cox and Plale, 2011). On the other hand, the filtering requires the definition of key words or hashtags to define the research and it has been argued that certain keywords or hashtags may not only give a partial data set related to a topic, but also could introduce a systematic bias. 
Moreover, a sample of Twitter users considered for a study does not represent the offline population nor all the Internet users. As it was shown in a survey carried out on May 2016 in the USA, only $24 \%$ of the people with internet access and $21 \%$ of the total population used Twitter (Greenwood et al. 2016). Moreover, it does not even represent all Twitter users because not all of them are interested in the same topics (Ahmed, 2015). This may lead to the exclusion of certain populations, generating biased results and new social discriminations and aggravating existing structural inequalities. In fact, the same survey showed that disadvantaged and elderly people continue to be underrepresented online as younger people were more likely to use Twitter than older people. Thirty six percent of online adults between 18 and 29 years of age are Twitter users, compared to $23 \%$ of people between 30 and 49, $21 \%$ between 50 and 64 and only just $10 \%$ of over 65 . Twitter was also more popular among the highly-educated ( $29 \%)$, better paid (30\%) and urban citizens (26\%) (Greenwood et al. 2016).

Furthermore, it is also difficult to obtain demographic data from tweets since users often do not provide this information. This is a great problem for social research because this kind of information is often vital to examine differential patterns in humans' behavior (McCormick et al. 2016). For these reasons, Tweets can be used for theory generation, but it must be clear that they are related to a specific respondent pool.

Other problems are that tweets are messy and unfocused data that do not respond to a specific research question, they can generate skewed results due unrelated information, they present an idiosyncratic nature and users appear multiple times in the data (McCormick et al. 2016). Moreover, as Marks (2013) demonstrated in his research "Happy, snappy tweets gain more followers", so people tend to tweet positive messages to be popular on Twitter, which means that more positive than negative tweets are posted. Additionally, Twitter gives information about what people do, but these activities are limited to those things that users like to share with their friends or followers. Similarly, it is possible to know when people write, but not when the activities they describe actually occur.

Finally, since most of the social researchers lack the skills to process online data, computer scientists are the ones that are taking advantage of this continuous flow of data. However, they do not always have the appropriate training to make the correct queries, to identify unsupported assumptions and to face the social consequences of the results (Asanet, 2012).

Besides these methodological problems, the use of tweets for research also implies ethical issues. Even if most tweets are considered public, since approximately $88.2 \%$ of the Twitter accounts are not protected, they are still linked to people's lives. Consequently, researchers must handle publications with care, particularly when they are related to sensitive topics (McCormick, 2015).

For these reasons, there is a huge controversy related to the use of tweets as data source for social research. Some people argue that widely available data, no matter the scale, are not better data and, therefore, are not an option. Others say that Twitter could be used as current and historical data source for social science research, but as a complement to traditional data sources (Asanet, 2012). Nonetheless, it must be accepted that Twitter provides important insights into human behavior and it should not be discharged as useless. Instead, researches must be creative and innovative in how to collect and analyze them in a proper way. 


\section{Concept and Methods}

\subsection{Aim and objectives}

In this context, this project aims to establish reference comfort ranges for weather parameters based on human responses to weather conditions extracted from tweets posted within the Northeast Climate Region of the USA during autumn.

The idea is to collect and store tweets that contain weather-associated keywords from the Twitter streaming API and then link them to real-time meteorological data collected close to the specific locations the tweets are posted. Afterwards, people's perception of weather will be extracted from the filtered tweets using a classifier trained specifically on weather data that will identify irrelevant, neutral, positive and negative tweets. Subsequently, positive and negative tweets and their related atmospheric data will be analyzed to establish comfort ranges for each weather parameter under study. Finally, the resulting ranges will be compared to other comfort ranges obtained in previous studies to evaluate the reliability of the Twitter stream for assessing human outdoor perception.

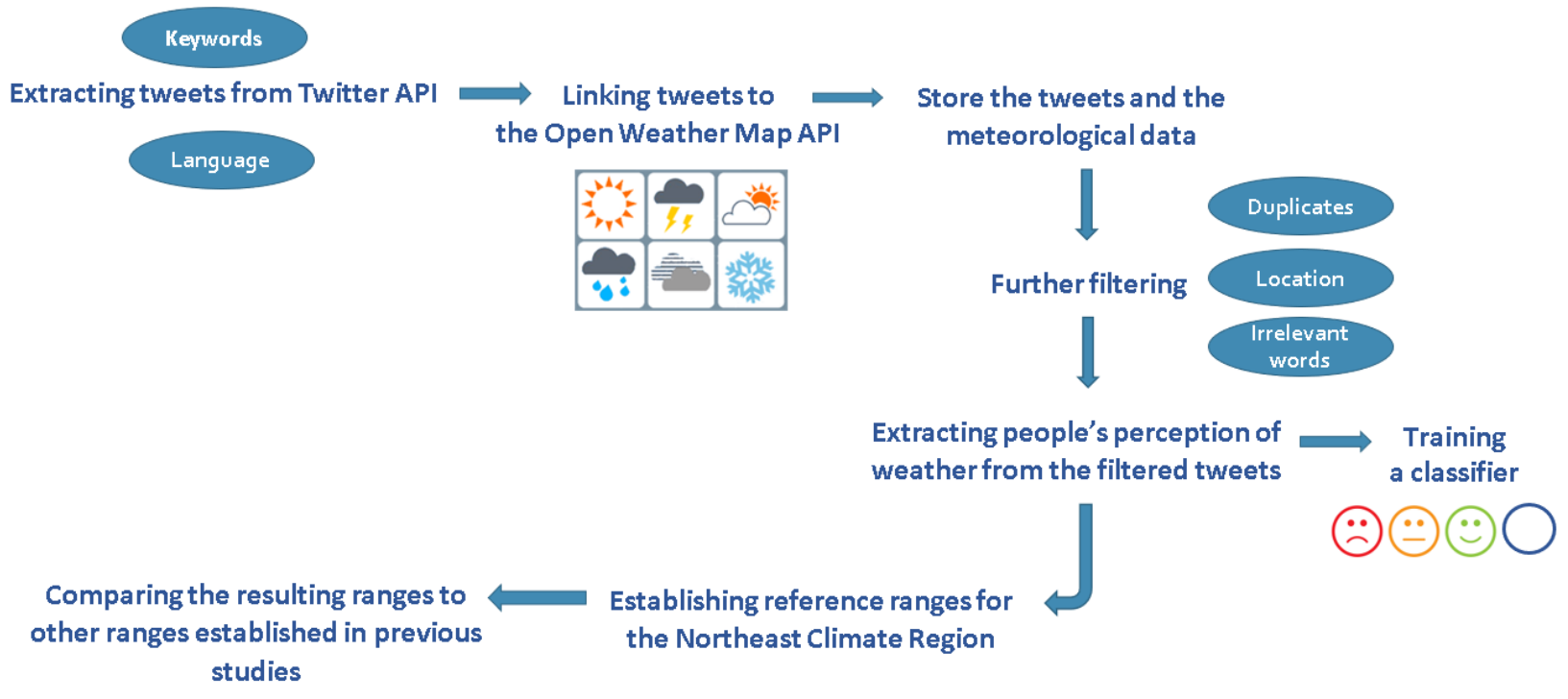

Figure 2. Concept and methods scheme

Since the influence of the adaptation factor must be considered, this project will address a specific location during a specific period. However, if the methodology works and thus Twitter is proved to be a reliable source of information for this purpose, future work could consider wider areas and times. 


\subsection{Study area}

The Northeast region of the USA was chosen as the study area for very specific reasons. First, The United States of America are the country with the highest amount of Twitter users, as it was illustrated by the social media update of May 2016 (Figure 3), 67.54 million people had an active Twitter account in the USA, representing $21 \%$ of the entire population. This means that this country is generally the most suitable one to evaluate the reliability of Twitter as a data source.

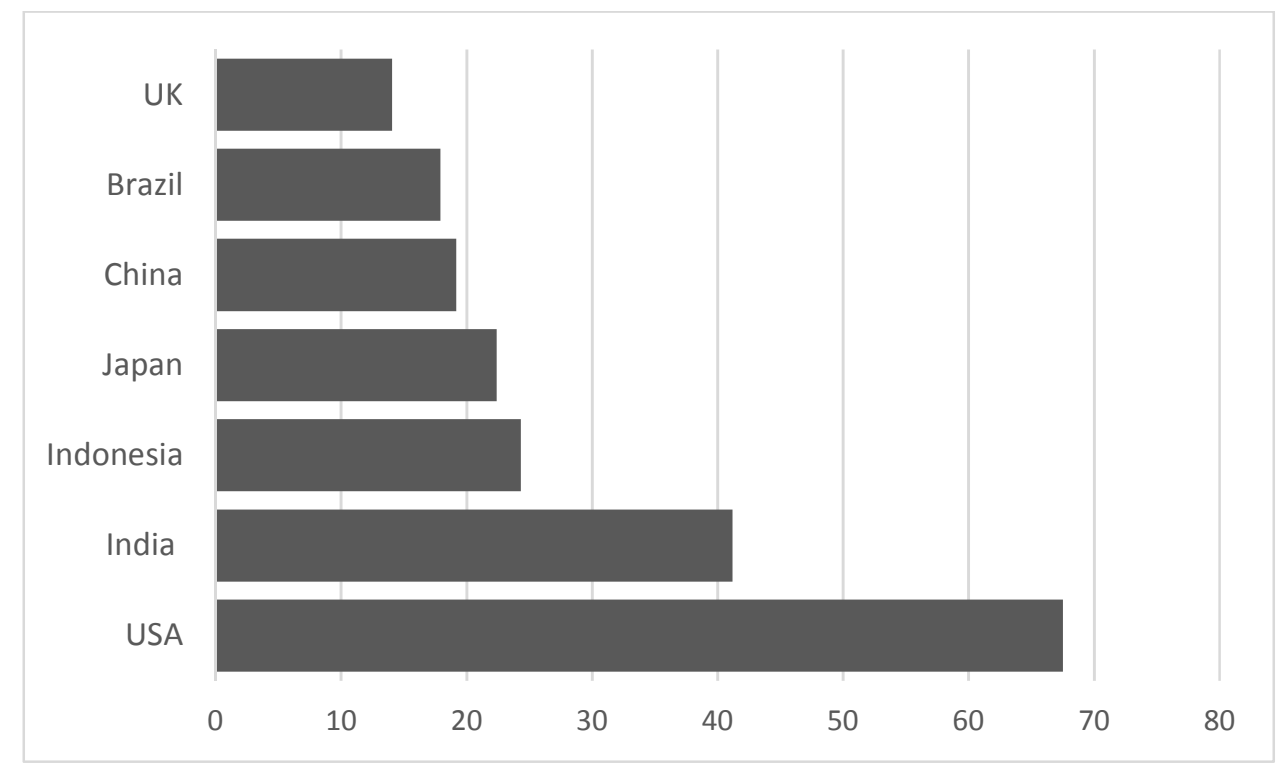

Figure 3. Number of active Twitter users in millions by May 2016. Source: Worldwide Statista market analytics

(Greenwood et al., 2016)

Secondly, a map regarding the distribution of weather tweets across USA was made, using the testing data collected during August 2016 (Figure 4). As it can be seen, the Northeast, the Southeast and the West regions showed the highest amount of weather tweets across the country. However, the Northeast region is the one with the highest weather variability over the seasons.

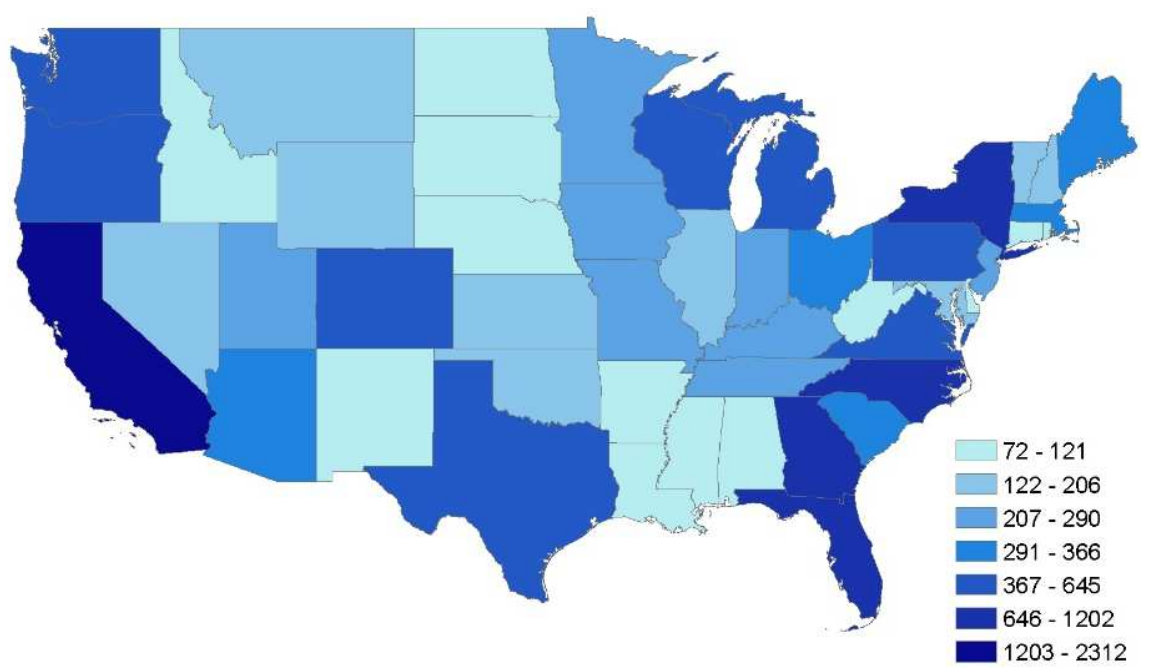

Figure 4. Number of collected tweets by state. August 2016. Source. Twitter.

The Northeastern United States, as defined by the U.S. Census Bureau, includes Maine, New Hampshire, Vermont, Massachusetts, Rhode Island, Connecticut, New York, New Jersey, and Pennsylvania. 
It has also been defined as an independent climatic region by the National Oceanic and Atmospheric Administration (NOAA) (Figure 5).

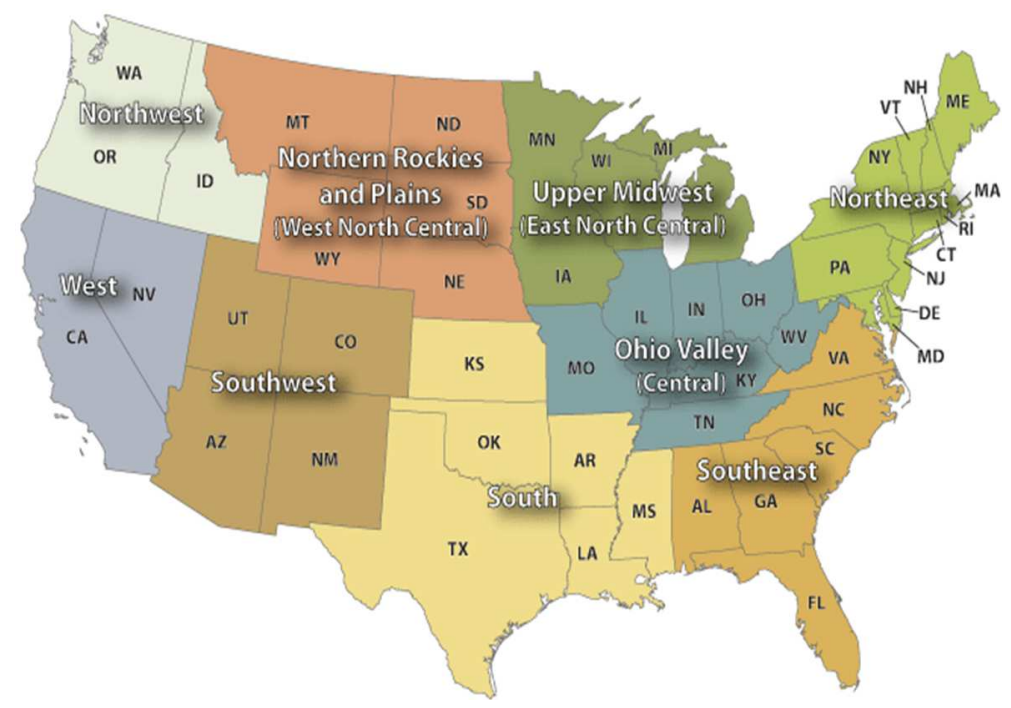

Figure 5. Climatic regions in USA. Source: www.ncdc.noaa.gov

The Northeast shows a varied climate. Average temperatures decrease towards the north, with distance from the coast, and in the mountains. Average annual rainfall varies by about $500 \mathrm{~mm}$ throughout the region, from $1270 \mathrm{~mm}$ annually in the coastal areas and elevations to $812 \mathrm{~mm}$ in Pennsylvania and New York. During winter, frequent storms bring cold and precipitation, especially to the north. Snowfall ranges from over 2500 $\mathrm{mm}$ per year in New York to small amounts in Maryland. Generally, it presents warm, humid summers and snowy, severely cold winters in the north and hot, humid summers and moderately cold, snowy winters in the south (NOAA, 2017). Finally, it is important to mention that the Northeast region belongs to the humid continental climate (Dfa-Dfb) in the climate classification made by Köppen.

This region is also one of the windiest in the USA, as Figure 6 illustrates.

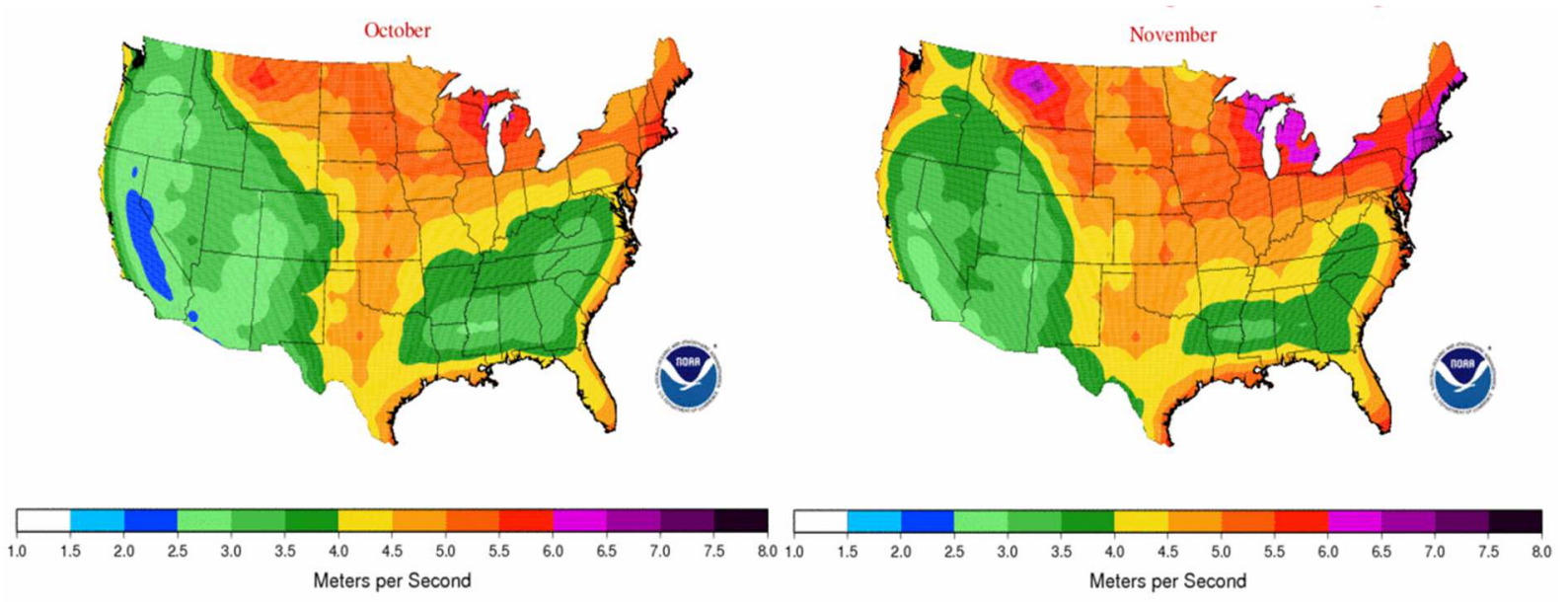

Figure 6. Wind speed during October and November 1971 -2000. Source: www.nrcc.cornell.edu 
The climate has been changing in the last years in the Northeast region (Figure 7) and records show earlier springs, hotter summers and warmer autumns and winters.
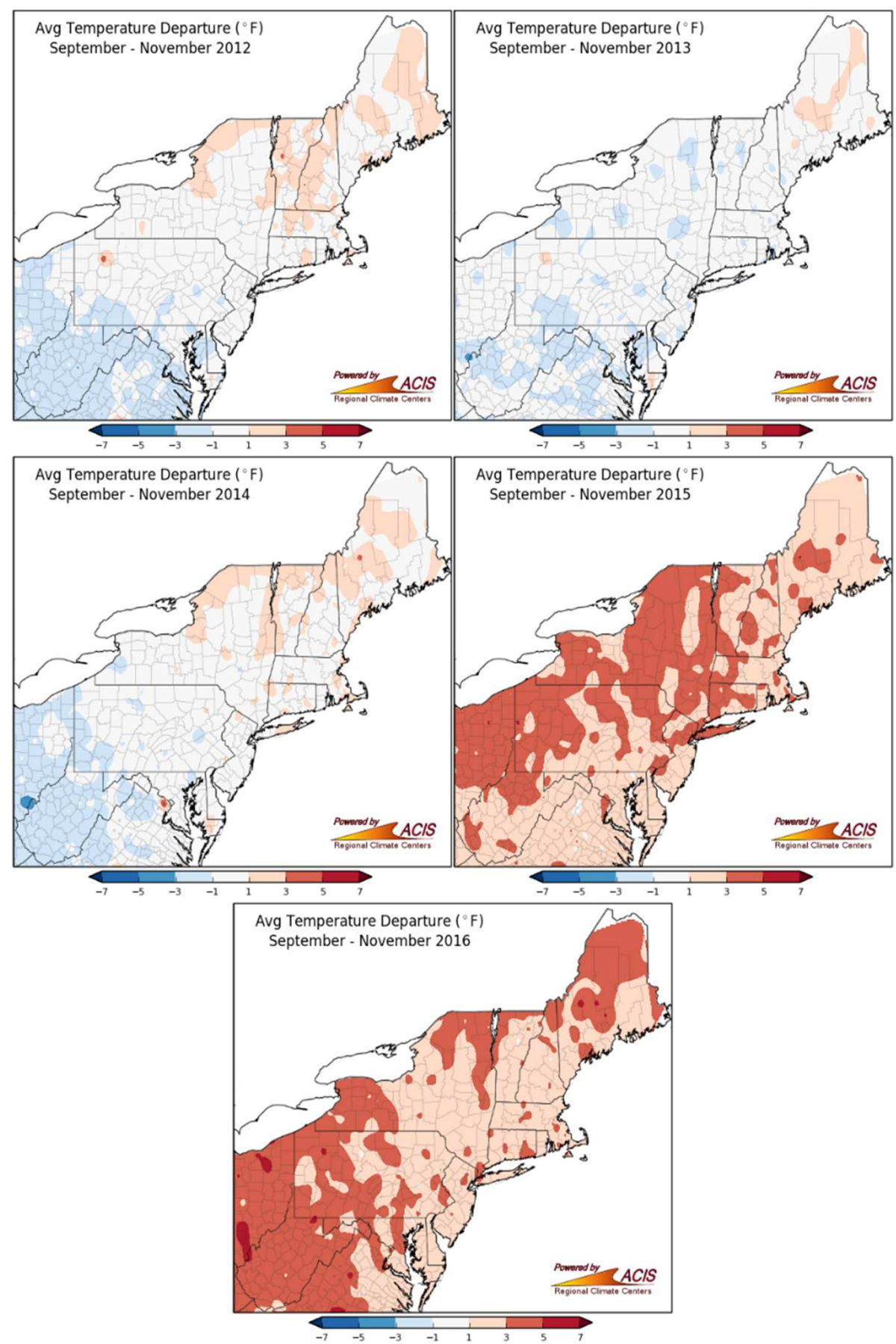

Figure 7. Average temperature departure. Autumn 2012, 2013, 2014, 2015, 2016. Source: www.nrcc.cornell.edu

In fact, in 2016, the region had its third warmest year on record. Regarding autumn, it has been showing higher temperatures and a three-week delay in the arrival of the typical cool fall weather. Also, short recurrences of warm summer days during the fall months called Indian summer (Figure 8 ) are becoming more frequent. The year 2016 had the third warmest autumn on record for the Northeast region. West Virginia had its second warmest autumn on record, while New York, Washington D.C. and Connecticut had their warmest autumn on record (NRCC, 2017). 
Concept and Methods

20

Nothing beats perfect Indian Summer weather, a day off \& a jog in the park past this place,...

instagram.com/p/BJ_TPyZhHgj/

11:19 PM - 5 Sep 2016 from South Park Township, PA

4 27

Figure 8. Tweet on Indian Summer. Source: https://twitter.com

18| P a g e 


\subsection{Data}

For this study case, two different kinds of data were collected. First, tweets related to weather that were posted within the Northeast region of USA during autumn and, second, real-time weather data linked to the locations of the tweets. In this chapter, the data sources will be introduced, the processes of data collection and filtering will be explained and the specific data fields that were used for the HCOS assessment will be illustrated.

\subsubsection{Data sources}

The collection of data from a web platform can be automated by using a program to access the platform's Application Programming Interface (API). In this way, data can be gathered thought structured queries regarding specific topics or attributes. For this project two APIs were used. The Twitter API and the Open Weather Map API.

\section{Twitter API}

The Twitter API includes the related documentation, data, code, and other materials that Twitter made available for developers to access its data. Twitter provides the REST API, in which a user can make one or more requests and then obtain the response, and the Streaming API that continuously deliver new responses to users' queries. Connecting to Streaming API requires a persistent HTTP connection. While the Streaming model is more complex than the REST model (Figure 9), it gives the possibility of having a real-time stream of Tweet data.

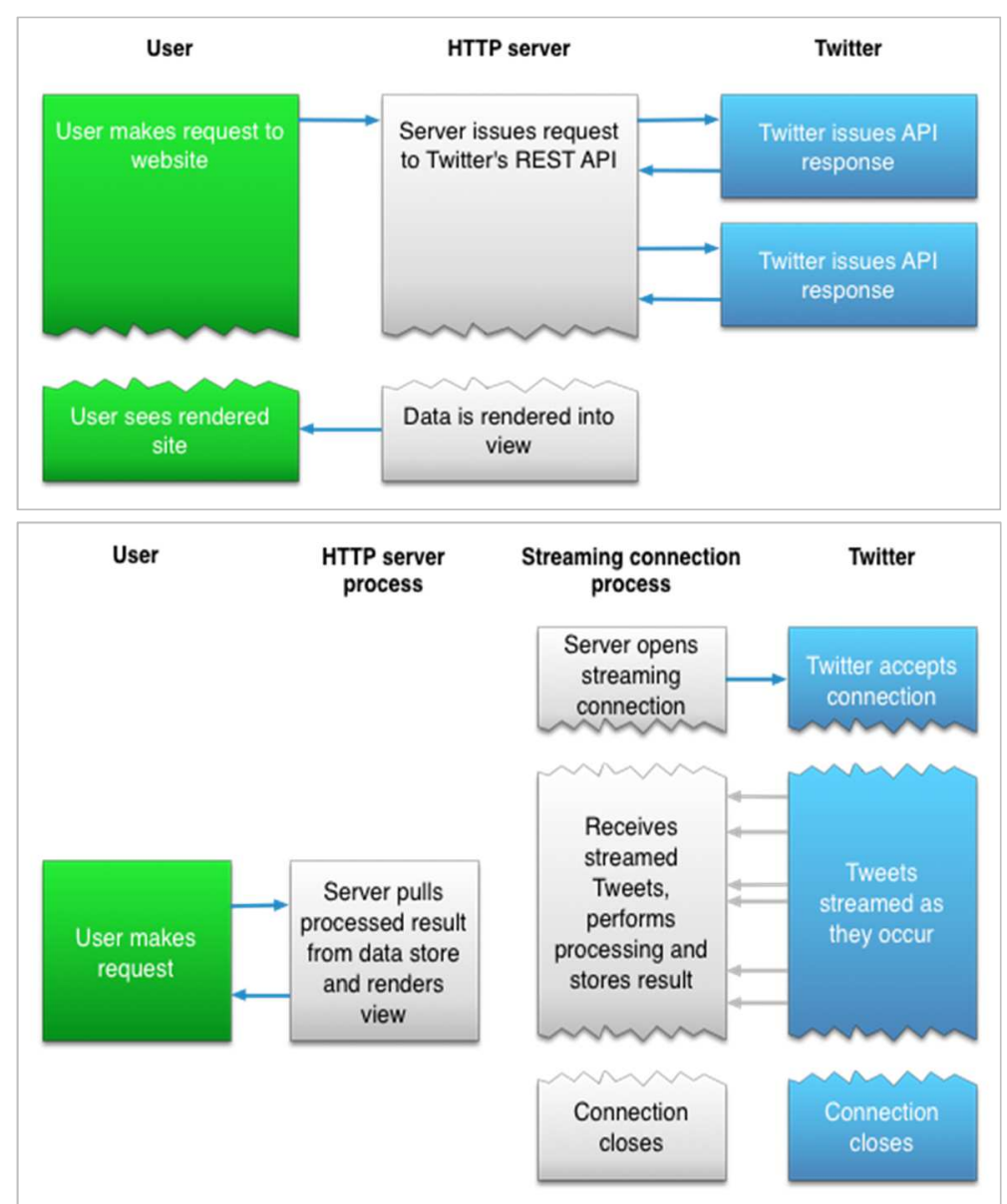

Figure 9. REST API model vs. Streaming API model. Source: https://dev.twitter.com 


\section{Open Weather Map API}

The Open Weather Map service allows to collect current and historical weather data, as well as weather forecasts. It is based on a spatial data science platform that collects, processes and distributes information. The collection of data includes more than 40.000 professional and private weather stations and forecasts of meteorological services and research laboratories. Long-term and short-term forecasts and real-time data from weather stations are combined and stored in the database and then processed with algorithms to create interpolated data of current weather conditions. Finally, the API allows access to data at any location on Earth, including over 200,000 cities, to support research and inspire new innovative applications. Data is available in different formats and calls can be made by geographical coordinates, by city ID or by ZIP code (Open Weather Map, 2012).

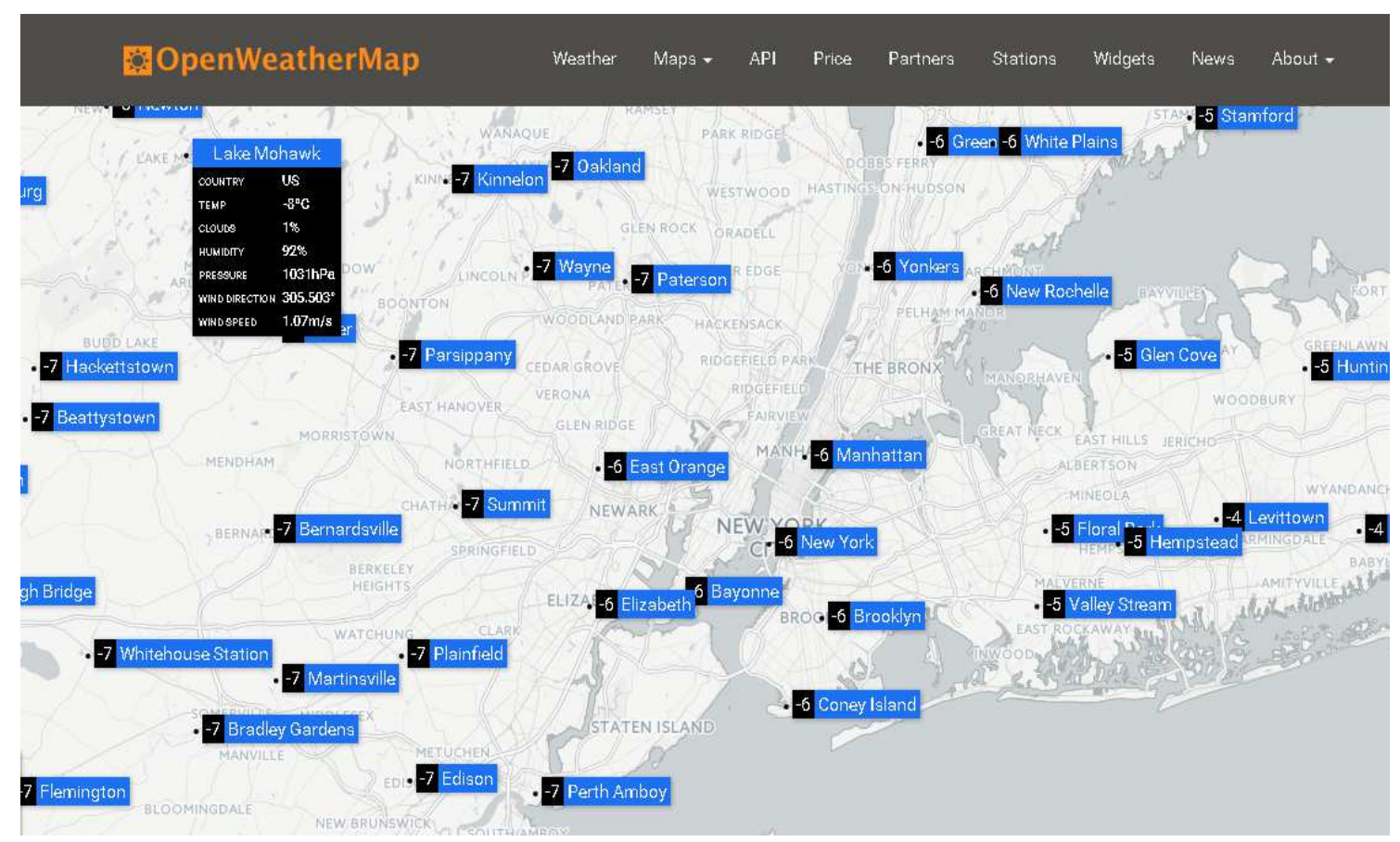

Figure 10. Open Weather Map weather stations in New York city. Source: https://openweathermap.org

\subsubsection{Data collection}

The process of collecting and cleaning data was divided into three stages. Extracting tweets from the Twitter API, linking tweets to the Open Weather Map API and storing the tweets and the meteorological data on the Raspberry Pi.

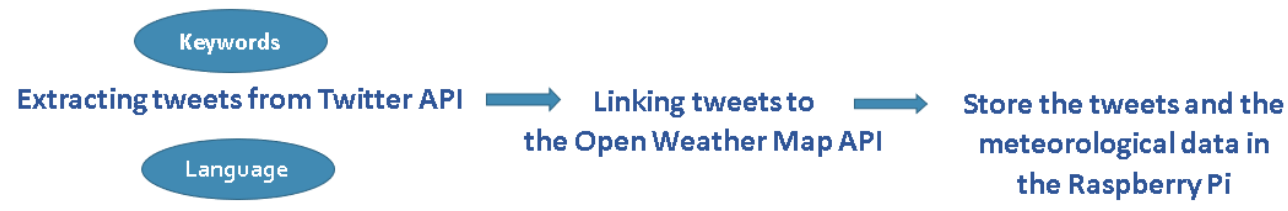

Figure 11. Data collection scheme

In this case study, the Twitter streaming API was accessed using a Python script and the tweepy library. Queries were designed to capture information on individuals who expressed their feelings or opinions regarding their weather perception. Only tweets written in English that contained any of the following key words, which were collected from several weather glossaries online, were used for the extraction stage: 
rain, rainy, wind, windy, sunny, sun, breeze, chilly, drizzling, flurries, fog, foggy, hail, frost, humid, humidity, hot, cold, temperature, blizzard, cloudy, heat, snowy, sunshine, thunder, umbrella, weather, storm, snow, snowing, raining, freezing, freeze, warm, mist, haze, ${ }^{\circ} \mathrm{F}$, downpour, blustery, gale, graupel, overcast, scorching, chill, dry, arid, sunscreen, sunburn, sunlight, sweltering, sizzler, autumn, summer, springtime, summertime, ${ }^{\circ} \mathrm{C}$, colder, coldest, hot, hottest, warmer, warmest, muggy and sleet.

For every received tweet, the script performed a selection in which only those that contained geographic information (coordinates) were chosen. Afterwards, for each chosen tweet, a call by geographical coordinates to the Open Weather Map API was made to obtain the current meteorological data for the tweet location. The resulting information was then stored in a CSV file.

The variables collected from the Twitter API were:

- User ID is a unique number that identifies each user.

- User name refers to the name of the user, as they defined it when the Twitter profile was created. It is not necessarily a person's name.

- Tweet text is the 140-characters text that compose the status update. It usually comes with a link to a picture or website.

- Tweet Time refers to the moment in which the Tweet was created.

- Tweet Coordinates represents the geographic location of the Tweet as reported by the user or client application. The inner coordinates array is formatted as GeoJSON (longitude, latitude).

- Tweet city, region and country are names of locations that correspond to the Tweet coordinates. They can be attached to Tweets by specifying a place id when tweeting.

The variables collected from the Open Weather Map API were:

- Temperature in Celsius $\left({ }^{\circ} \mathrm{C}\right)$

- Wind speed in meter per second $(\mathrm{m} / \mathrm{s})$

- Relative Humidity in percentage (\%)

- Cloud coverage in percentage (\%)

- Weather description

To maintain the constant HTTP connection required by the streaming API and store the stream of tweets, a Raspberry Pi was used. It is a low-cost, high-performance and credit-card sized computer (30).

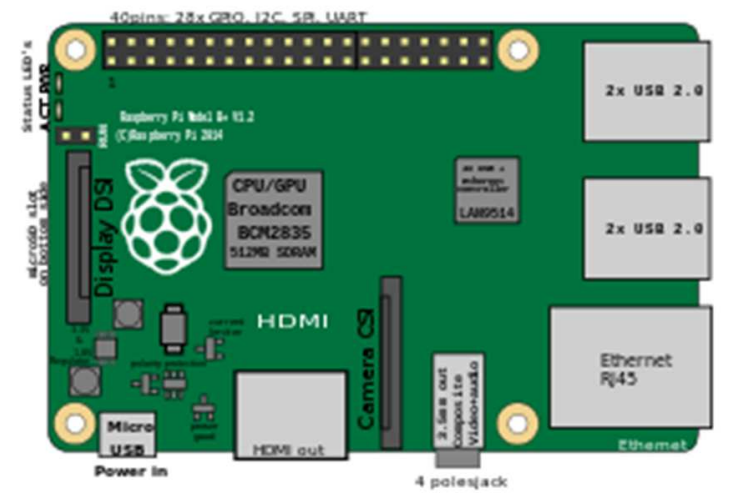

Figure 12. Raspberry Pi. Source: www.raspberrypi.org/about 
Once the connection was set, 1.198.663 tweets were collected from September 1th to November 31th. From those, 201.580 were posted within USA and 38.780 within the Northeast region.

\subsubsection{Further Filtering}

After the extraction of the data, further filtering had to be made to clean up the collected data. The first step was to eliminate duplicates. Then, only tweets posted within the Northeast Region of USA were selected.

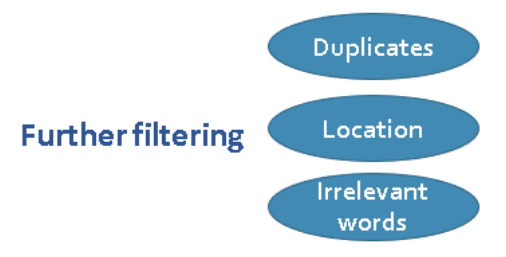

Figure 13. Further filtering scheme

Afterwards, potentially irrelevant tweets were filtered out to effectively capture peoples' weather perception. This required a methodical read of the tweets aimed at creating a second list that included frequently-occurring irrelevant words. These words were mostly related to weather reports, as it can be seen in Figure 14.

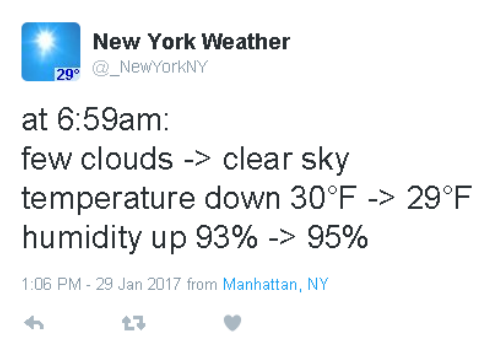

Figure 14. Tweet example of weather reports. Source: https://twitter.com

Finally, these tweets-which compose a great part of the total body of data collected-were systematically removed, along with job offers, products discounts and specific irrelevant hashtags, as they do not express any opinion or emotion and, therefore, are irrelevant for the perception extraction. The following is the list of irrelevant words:

"wind:calm", "humidity up", "humidity down", temperature up", "temperature down", "dew point", "today's records", "trump in", "good morning\#", "\#drinking a", "gusting", "\#I'm at", "USGS", "today's forecast", "barometer", "weather now", "hiring", "can you recommend anyone for this job", "diabetic", "Pittsburgh International Airport" and "just posted a photo@". 


\subsection{Perception extraction}

Sentiment analysis is the task of extracting the opinions, feelings or perceptions of authors from text. The approaches to sentiment analysis can be divided into two main groups, supervised and unsupervised.

First, the unsupervised approach is based on the definition of the semantic orientation (SO) of an object, namely sentences or words. To estimate its SO, an object is compared to two other sentences or words carrying opposite polarities. An example for this are words like good and bad. Based on their co-occurrence in a specific corpus of documents or on-line resources, the statistical dependency between them is calculated. Thus, it can be known if the given object is closer in meaning to the positive word (good) or to the negative (bad). Sentiment lexicons are used to give the syntactic, semantic, and pragmatic information required to estimate the co-occurrence of objects and, therefore, calculate their statistical dependency (Feldman, 2013).

Sentiment lexicons can be acquired in three ways. The first is building them by hand, which is a very timeconsuming process. Secondly, lexicons can be based on dictionaries like Wordnet because they are "large lexical databases of English nouns, verbs, adjectives and adverbs are grouped into sets of cognitive synonyms and interlinked according to conceptual-semantic and lexical relations" (Princeton, 2017). Finally, if a domainspecific sentiment lexicon has to be created, a corpus-based algorithm can be used. These algorithms generate graphs of words clustered by their function and polarity using the relations obtained from the corpus analysis.

Second, the supervised approach requires a set of training data that are already labeled. This means that part of the data is related to a set of classes that could contain either distinct emotions (anger, happiness, sadness) or polarity categories (positive, negative, neutral). The seeds are used to feed a classification (e.g. Naïve and Byes) or propagation algorithm (e.g. MAD) that classifies the rest of the data (Feldman, 2013).

Sentiment analysis can be applied at different levels: document-level, sentence-level and aspect-based. Giving the character limitations of tweets, applying sentiment analysis to Twitter data occurs on sentence-level.

When working with sentence-level sentiment analysis, it is important to distinguish the objective sentences from the subjective ones, because only the firsts contain opinions and feelings and, therefore, can be used for sentiment analysis. For this reason, supervised approaches for sentence-level sentiment analysis usually include a subjectivity classification, before applying the polarity classification. Moreover, one of the assumptions of the sentence-level sentiment analysis is that each sentence must express a single opinion, otherwise, they must be separated into parts until this requirement is met. Nevertheless, specific kinds of sentences, such as conditionals or sarcastic remarks, are exceptions and must be handled with special strategies.

It is important to consider that supervised approaches require a smaller number of seeds than unsupervised approaches to obtain the overall sentiment of the sentence. For this reason, supervised approaches are very common in sentence-level sentiment analysis.

Sentence-level sentiment analysis is a very complex process. Additionally, the nature of tweets, including very informal language, abbreviations, lack of orthography, slang words (Figure 15), introduces further difficulties (Kouloumpis et al., 2011). 


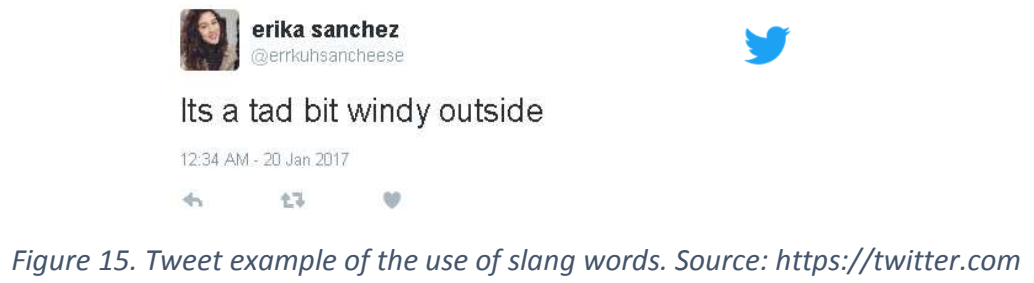

For this reason, some researchers have created methodologies that combine supervised and unsupervised approaches to improve their outcomes.

For example, in the Urban Emotions project (Resch et al., 2015), researchers used tweets to detect specific emotions. They decided to apply the Modified Absorption Algorithm (MAD), which is a graph- based semisupervised learning algorithm and requires only a small set of training data. MAD requires several steps. First, the tweets were filtered by language (English) and then volunteers were used for the annotation stage. Afterwards, the seeds were selected according to the emotions they contain. Subsequently, the pre-processing stage started. In this case, the researchers used part-of-speech tagging and a lexicon called ANEW. Next, the degree of similarity between labeled and unlabeled tweets was calculated. For Urban Emotions, the similarity was not only based on linguistics and semantic content, but also on temporal and geospatial distance. At this point, they built the graph. The seeds and the unlabeled data were the nodes and the edges between them described their similarities. The stronger the edge, the higher the similarity degree. Finally, MAD was applied to the graph. It propagated the labels of the seeds over the unlabeled tweets using the similarity scores. This was done by comparing the scores between all pairs of nodes, starting with the strongest edges.

Similarly, other researchers are using microblogging features, such as emoticons and hashtags (Figure 16), to improve the selection of the seeds and apply machine learning techniques.

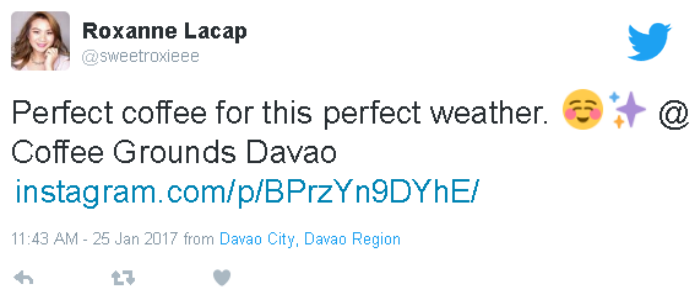

Figure 16. Tweet example of the use of emoticons. Source: https://twitter.com

For example, Go et al. (2009), applied the Naïve and Bayes algorithm, which is a probabilistic classifier based on the application of the Bayes theorem. This theorem describes the probability of an event in compliance with prior knowledge of the conditions that are related to that event. In this case, the prior knowledge was represented by the seeds including different polarity emoticons. They reached $81 \%$ accuracy on their tests, but also found that the accuracy of this method decreases when using three categories (positive, negative and neutral), instead of two (positive and negative).

In another study (Hannak et al., 2012), decision trees, which are machine learning techniques, were applied to a general Twitter corpus (not weather-related) to predict the aggregate sentiment given the time of the day, the season and the weather. They constructed a Twitter-specific token list that used positive and negative emoticons and calculated the relative fraction of times that each token occurred with a positive or a negative emoticon. Afterwards, they were able to estimate the sentiment score of each tweet. Nevertheless, they only obtained $65 \%$ accuracy when comparing their sentiment analysis methodology with human ratings collected on Amazon Mechanical Turk. 
Furthermore, several classifiers are available online to perform sentiment analysis due to the increasing demand of this service. The AYLIEN Text Analysis API was tested using weather-related tweets. AYLIEN is described as a "package of natural language processing, information retrieval and machine learning tools for easily extracting meaningful insights from textual content" (Aylien, 2012). It had a very high accuracy regarding the classification of positive, negative and neutral tweets, but it could not identify tweets irrelevant to the weather perception analysis, as AYLIEN was not trained on weather related data, but on general topics.

In a nutshell, it can be said that traditional unsupervised approaches are suitable for the present study case, because they allow the creation of domain-specific sentiment lexicons. Nevertheless, this task is very complicated and requires a great deal of time. Similarly, semi-supervised approaches can also fit the aim of this project because they need a small set of seeds and could use already created lexicons. Unfortunately, a weather perception lexicon was not found online. Moreover, they require the creation of a similarity-based graph that would exceed the time and the effort available for this thesis. On the other hand, there are several services available online that offer sentiment analysis based on machine learning techniques, which fall into the category of the supervised approaches. Nevertheless, to completely fulfill the requirements of this thesis, the classifier must give a highly accurate performance on weather data, distinguishing between relevant and irrelevant tweets. Additionally, it must be trained to identify neutral, positive and negative tweets, because neutral tweets do not express opinions (Figure 17) and, therefore, cannot be used to extract people's perception of weather.

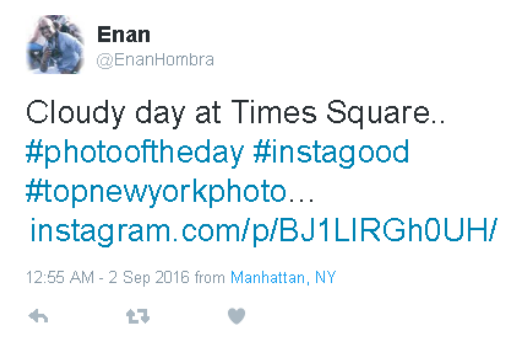

Figure 17.Neutral tweet example. Source: https://twitter.com

With all this in mind, training a weather-specific classifier that can distinguish among irrelevant, positive, negative, and neutral tweets was considered as the most suitable option for the aim of this thesis.

\subsubsection{Training a weather-specific classifier}

To train a domain-specific classifier, three stages were completed. The preparation of the training data (also called annotation), the creation and training of the classifier and the evaluation of the results.

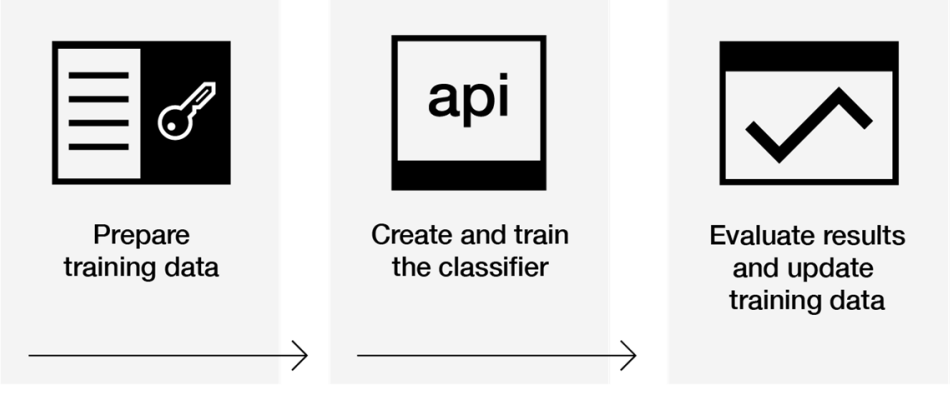

Figure 18. Using the Natural Language Classifier service. Source: www.ibm.com/watson

The first step is the annotation. It entails the collection of seeds or labeled data to feed the classifier. 
In the last years, platforms like Amazon's Mechanical Turk and Crowdflower have been offering the possibility of using crowdsourcing to annotate texts to be used for natural language classifications. This methodology usually has better results on the annotation stage than part-of-speech and sentiment lexicons. Humans can easily recognize and understand sarcasm and irony, misspellings, slang words, abbreviations and other features that are distinctive of the microblogging nature (Crowdflower, 2017). Additionally, these platforms offer the possibility of collecting several judgements, made by different people, for each tweet, giving a measure of accuracy to each annotation. Tweets with a low accuracy percentage can be filtered out, increasing the quality of the final set of seeds. Seed selection is an important step because it strongly influences the output of the sentiment analysis process.

For the present thesis Crowdflower was selected because it allows a better quality control of the annotations than Mechanical Turks. For example, the user can identify and exclude unreliable annotators based on tests that he or she can design based on the aim of their research project (Nakov et al., 2016). It also gives a final report including all the annotators that contributed to a specific work and the demographic information related to them. Moreover, the use of Amazon's Mechanical Turk has been restricted to US residents.

Crowd flower is easy to use. The platform provides specific templates that respond to the user's necessities. It also allows the customization of the task: the user can explain the goal of the research and give detailed instructions to the annotators. As mentioned above, the platform asks for a set of test questions that can be used to evaluate the performance of the annotators. After launching the project, it is possible to monitor the collection of judgements. At the end, the results are automatically compiled, stored and aggregated, if required.

For the assessment of the HCOS, 15.000 tweets were annotated with 2 judgments each using Crowdflower. An additional third judgement was added manually. The annotators first decided whether a tweet was relevant or not, and if relevant, put the tweet in one of the three categories: positive, negative or neutral. Only those tweets in which two out of three judgments agreed were used as seeds. The tweets selected as seeds were equally distributed over the three months of the study period (5000 per month). This allowed the inclusion of a greater range of expressions and vocabulary related to the variation of weather over the months.

Once the seeds were ready, they were used to train the so-called Watson Natural Language Classifier, which is a service offer by IBM. Watson can be trained to learn predefined classes in example texts and then apply those classes to new inputs by using a mathematical approach called deep learning. It can be said that Watson mimics the human ability to classify phrases into categories by understanding the intent behind text (IBM, 2017).

To successfully train a natural language classifier, each text value and class in the training data must represent the kinds of texts and classes that the classifier is expected to predict. Moreover, the classes that are associated with each text must be correct. Finally, the more training data, the more accurate are the results. Consequently, the trained classifiers can be only as good as the training data.

The main reason Watson was selected for this thesis was that it allows the creation of natural language classifiers without prior knowledge in machine learning. The input of the service is the training data and the outputs are the corresponding classification and the associated confidence levels. Also, Watson works best with short texts, which makes it perfect for tweets analysis, and it can also be trained for a specific domain. 
Four different classifiers were created and trained. One included the whole season under study (autumn), while the other three included only one month each (September, October, November). The reason behind this choice was to evaluate the accuracy of them, compare the results and then select the most accurate alternative.

The autumn classifier was initially trained with the high accuracy seeds obtained from Crowdflower. Then an additional set of seeds was added to complete the 150000 that Watson permits. The monthly classifiers, on the other side, were fed with only 5000 seeds each. Tweets from September were used to feed the September classifier, Tweets from October were used to feed the October classifier, and so on.

Finally, the autumn classifier was used to classify the rest of the data because of its higher accuracy (90\%), since the monthly classifiers, altogether, were less accurate (86\%). 


\subsection{Analysis}

Two aspects must be taken into consideration before beginning the analysis. First, after the perception extraction process, 13614 relevant tweets were collected: 3425 were neutral, 6483 positive and 3706 negative. This confirms the general tendency of social media posts, showing more positive than negative tweets (chapter 3). In this case, however, there could be an additional explanation for this positive attitude related to what Palutikof et al. (2004) discovered when studying people's responses to exceptional mild weather in Great Britain. Sixty six percent of his respondents declared to prefer unusual warm weather than unusual cold weather and the idea of having milder winters and autumns was perceived as positive or very positive. And, as was mention in chapter 4.2, the northeast region had its third warmest autumn on records (Figure 19).

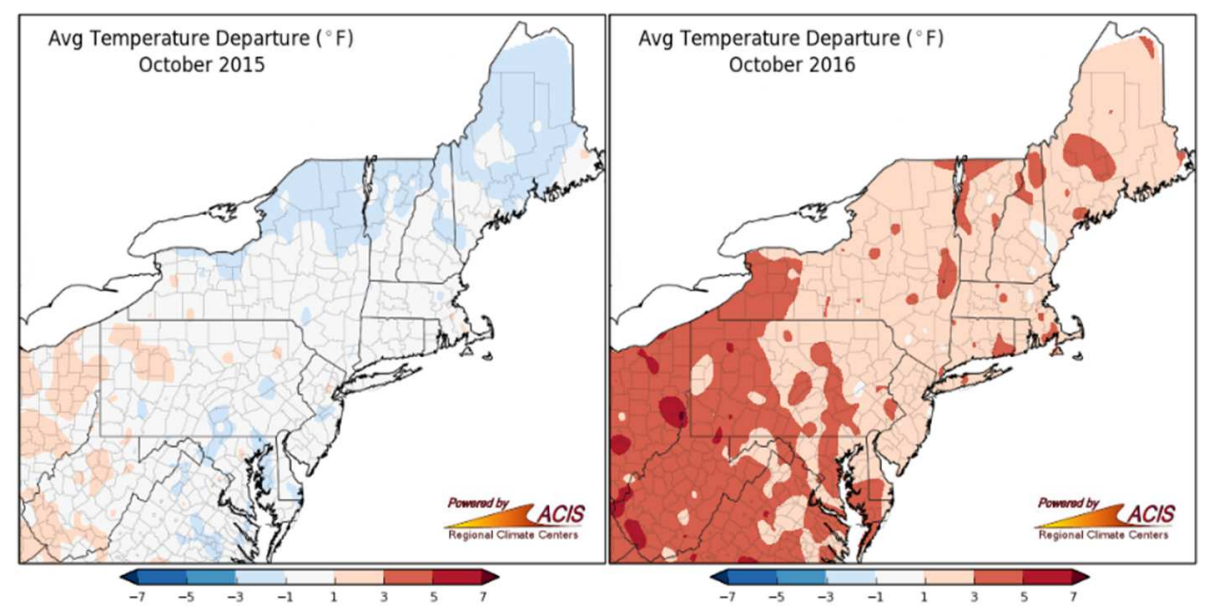

Figure 19. Average temperature comparison between October 2015 -2016. Source: www.nrcc.cornell.edu

Due to the different amount of positive and negative tweets, the results were normalized before being analyzed, with the exception of those that show absolute numbers of collected tweets. The weather parameters where divided into classes, then the number of positive and negative tweets in that class was normalized by the total number of tweets in that class. This kind of normalization allows to compare positive and negative tweets within a certain range of values and, to build stacked column graphs allowing the direct overview of the distribution of the tweets.

Another consideration before the analysis must be made regarding the weather parameters collected for this thesis. In chapter 4.2, the collected fields were shown. They included temperature, wind speed, relative humidity, cloud coverage and weather description, but not solar radiation. Even if this last parameter was proven in previous studies to be very important for the assessment of HCOS, it was not available for all tweets because not all the weather stations in the Open Weather Map platform measure it.

Since the aim of this work is to define comfort ranges for HCOS using tweets and then compare them to ranges that were established in previous studies, the first step was to decide what kind of ranges had to be defined. As it was mentioned before, HCOS ranges can be related either to indices, that combine 2 or more weather parameters, or to single weather parameters. In this context, ranges were established for the Effective Temperature index (ET) and the Thermohygrometric Index (THI), as well as for temperature ( $t$ ), relative humidity $(\mathrm{RH})$, wind speed $(\mathrm{V})$ and cloud coverage $(\mathrm{CC})$. In the next paragraphs the reasons for this choice will be discussed. 
In chapter 2, a list of simple indices (based on environmental variable measurements) and their characteristics was shown. There are several simple indices, but only Effective Temperature (ET) presents the suitable characteristics for this research: it is applicable to the wide range of thermal conditions, which is required for the analysis of the Northeast region of the USA. Additionally, it shows a high correlation with the indices that included thermo-physiological parameters, which are more accurate. ET considers wind speed $(v)$, humidity $(\mathrm{RH})$ and temperatures $(T)$, but not solar radiation into its formula, which for this thesis was not collected.

It has also been said in chapter 2 that there are two ways of estimating effective temperature. The first one is through the formula established by Missenard (Equation 1) that includes wind (v), humidity (RH) and temperature $(t)$ and which focuses on the cooling effect of wind on the thermal sensation (5). The second one is through Thom's THI formula (Equation 2) that includes only humidity $(\mathrm{RH})$ and temperature (t) and, therefore, shows the heating effect of humidity on thermal sensation (25). In this thesis, both comfort ranges, ET and THI, were evaluated.

$$
\begin{gathered}
E T=37-\frac{37-t}{0.68-0.0014 \times R H+\frac{1}{1.76+4 v^{0.75}}}-0.29 \times t \times(1-0.01 \times R H) \\
T H I\left({ }^{\circ} \mathrm{C}\right)=t-(0.55-0.0055 R H) \times(t-14.5)
\end{gathered}
$$

Furthermore, five weather parameters (temperature, wind speed, relative humidity, cloud coverage and weather description) were analyzed separately to identify the resulting comfort ranges and then compare them to the ones obtained by Sasaki et al. (2000). Among all the previous studies that were taken into consideration, Sasaki's work was selected for two reasons. First, they included the same weather parameters that were collected for this work, temperature, relative humidity and wind speed, as well as weather description. Secondly, according to the Köppen classification, Northern Japan and the Northeast Region of the USA belong to the same climatic region, the humid continental region (Figure 20). This means that the climate conditions are very similar and acclimatization effects can be neglected.

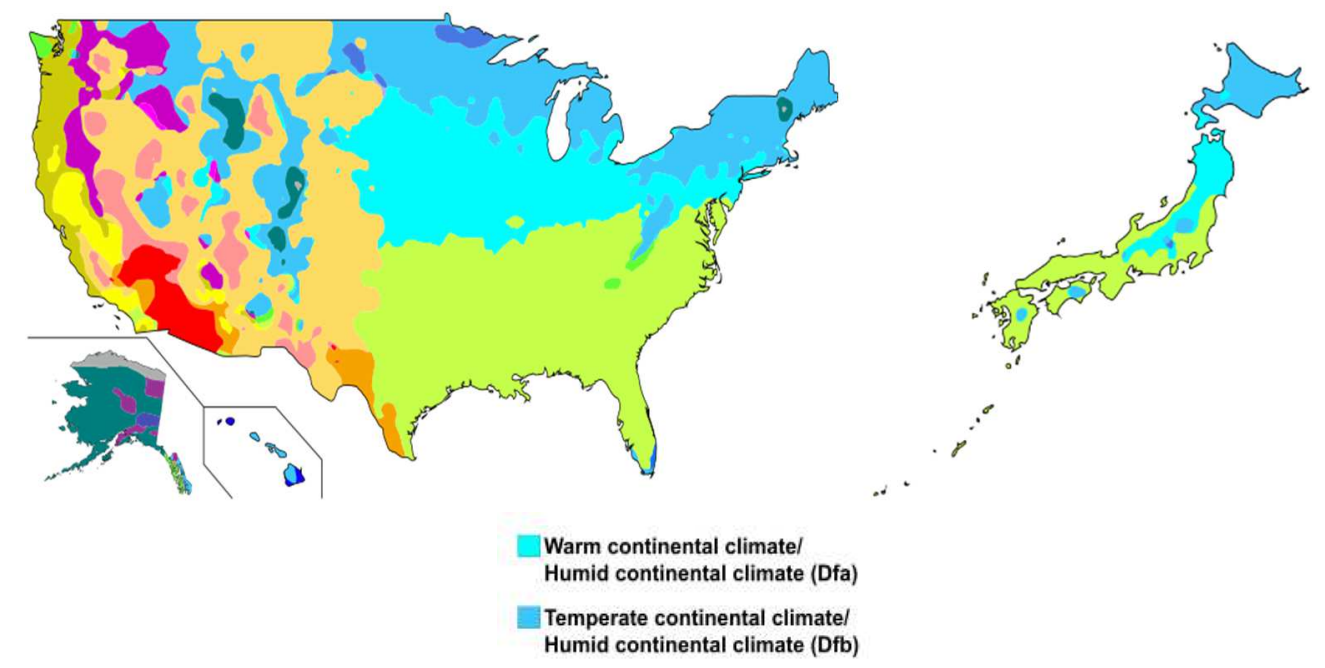

Figure 20. USA and Japan according to Köppen classification by Ali Zafan. Source: Wikimedia Commons 
In addition to Köppen's classification, the following climate profiles illustrate the similarities between the city of Aomori, in which Sasaki's research was carried out and the cities of Boston, New York, Pittsburg and Philadelphia, located within the Northeast region of USA.

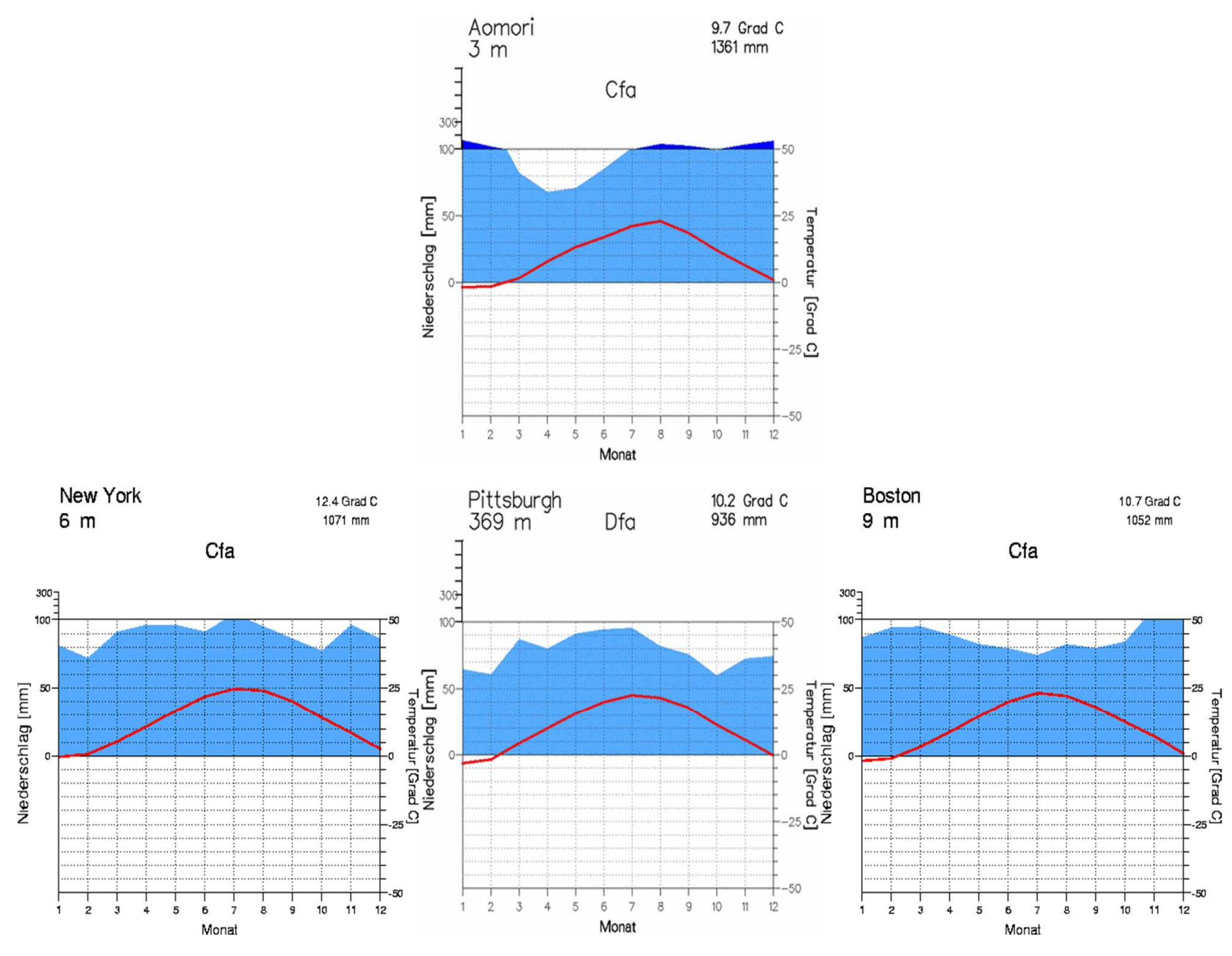

Figure 21. Climate diagrams comparison. Aomori, New York, Pittsburg and Boston. Source: www.klimadiagramme.de

As one may see in all the profiles, temperatures have the same tendency during the year, showing minimums of the monthly means at the extremes (around $0^{\circ} \mathrm{C}$ ) and maximums between July and August (around $25^{\circ} \mathrm{C}$ ). Regarding the precipitation, all these cities seem to be humid as their average monthly rainfall is always above $60 \mathrm{~mm}$ and the precipitation line is above the temperature line in the standardized climate diagrams of Figure 21. Nevertheless, the American cities present a higher variability than Aomori, which seems to be the most humid throughout the year with exception of the spring. 


\section{Results and Discussion}

In this chapter, the resulting ranges will be presented and then compared to the ones established by previous studies. Moreover, the current methodology will be compared to the proposed methodology.

\subsection{Indices}

\section{Effective Temperature}

The EF chart (Figure 22) illustrates the variation of weather perception with EF values. Several features can be identified from this figure. First, the percentage of positive tweets has a clear peak at ET values ranging from $14{ }^{\circ} \mathrm{C}$ to $16{ }^{\circ} \mathrm{C}$. This indicates that most people feel comfortable or happy when combinations of wind speed, humidity and temperatures are within this range. Moreover, this percentage shows a fast decrease when ET is lower than $14{ }^{\circ} \mathrm{C}$ and higher that $16^{\circ} \mathrm{C}$. Finally, there is a second peak at $-12{ }^{\circ} \mathrm{C}<\mathrm{ET}<-10^{\circ} \mathrm{C}$, which may need further exploration.

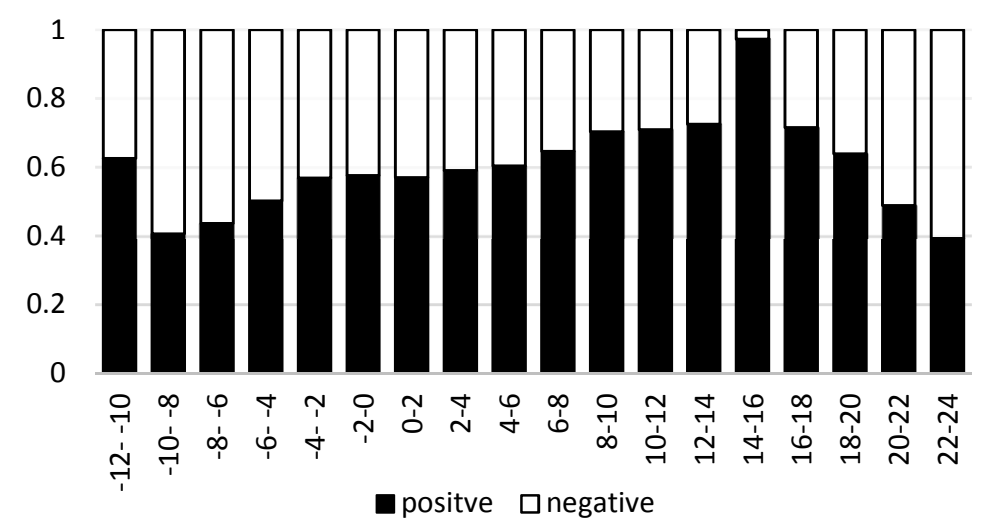

Figure 22. Effective Temperature variation from Twitter data

Thermal indices have specific assessment scales. In the case of ET, however, several assessment scales have been adapted for it. The thresholds presented in this work are the ones in use in Central Europe, where the "comfortable" range varies between $17^{\circ} \mathrm{C}$ and $21^{\circ} \mathrm{C}$ (Table 4).

Table 4.Effective Temperature ranges and assessment scale.

\begin{tabular}{|c|c|}
\hline ET & Thermal sensation \\
\hline & Sweltering \\
\hline$>27$ & Very hot \\
\hline $23-27$ & Hot \\
\hline $21-23$ & Warm \\
\hline $17-21$ & Comfortable \\
\hline $9-17$ & Cool \\
\hline $1-9$ & Cold \\
\hline$<1$ & Very cold \\
\hline & Frosty \\
\hline & Extreme hazard \\
\hline
\end{tabular}

When applying these thresholds to the Twitter data and comparing them to the obtained results, it becomes apparent that the latter are shifted to the left (Figure 23), since more people declared to be comfortable when the values of ET ranged between $9{ }^{\circ} \mathrm{C}$ and $17^{\circ} \mathrm{C}$, with a peak between $14^{\circ} \mathrm{C}$ and $16^{\circ} \mathrm{C}$. 


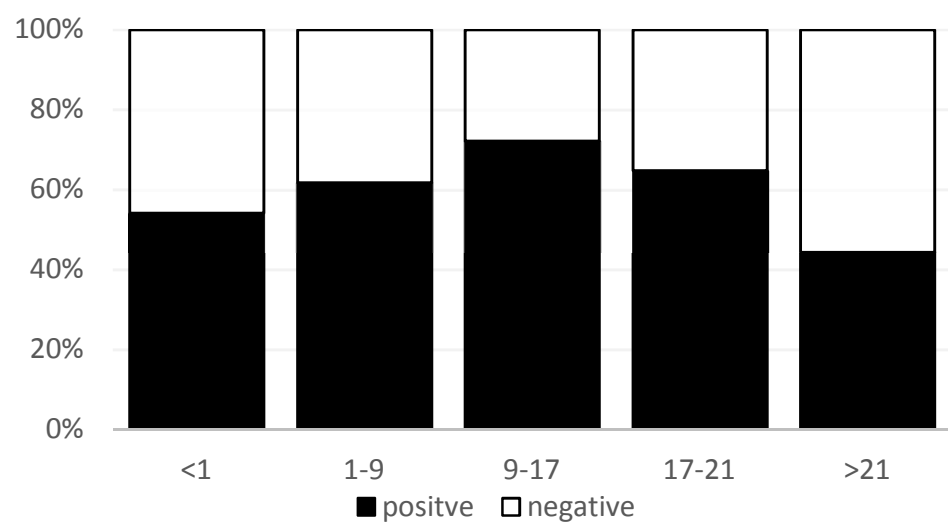

Figure 23. Effective Temperature ranges applied to the Twitter data

This shift may be explained by the following reasons. First, Central Europe and the Northeast region of the USA belong to different climatic regions. As it was mentioned before, acclimatization can influence people's perception due to adaptations to local weather. The region under study is one of the windiest of the USA (include values) and its inhabitants might have developed a wind tolerance. In fact, in another study (24), carried out from August to October in Nigeria, the researchers concluded that "the most appropriate comfort limits for the Effective Temperature Index in Zaria are 20-25 degrees Celsius", which indicates that ranges can vary from region to region.

Secondly, cultural differences between the USA and the countries within Central Europe can be accounted for skewed results, as Thorsson et al. (2007), de Montigny et al. (2011) and Palutikof et al. (2004) concluded in their studies. Also, it is important to consider that these thresholds were built on yearly data, while this thesis considered only autumn data. In this regard, Baranowska and Gabryl (1981) developed a more specific ET scale (figure 24), which is widely recognized as the most precise in reflecting seasonal variability of climatic conditions. From this scale, it can be concluded that comfort assessment scales that consider data from all the seasons cannot be applied to specific seasonal data, because they do not reflect the variations of the comfort sensation over the seasons in their ranges.

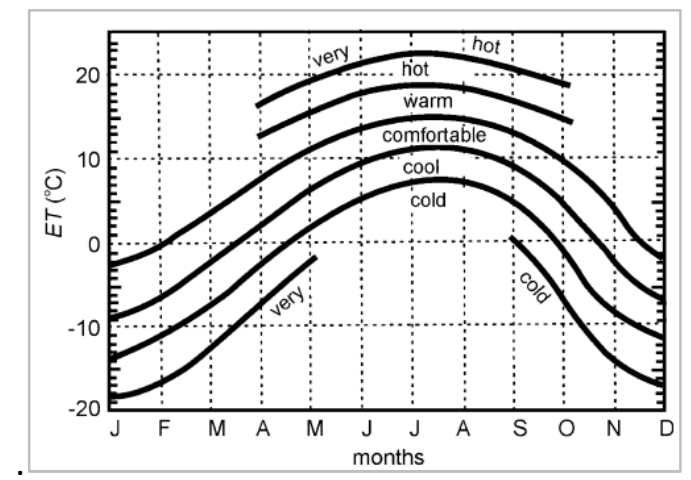

Figure 24. Effective temperature thresholds at different months. Source: BARANOWSKA and GABRYL, 1981.

\section{Termohygrometric Index}

The THI illustrates the variation of weather perception using THI values. Some tendencies can be highlighted from figure 25 . First, the percentage of positive tweets is highest at $\mathrm{THI}$ ranging from $22^{\circ} \mathrm{C}$ and $24^{\circ} \mathrm{C}$. Even if this peak is not clearly discernable from the rest of the ranges, slight decreases in the comfort percentages can be noticed when $\mathrm{THI}$ is lower than $22^{\circ} \mathrm{C}$ or higher that $24^{\circ} \mathrm{C}$. 


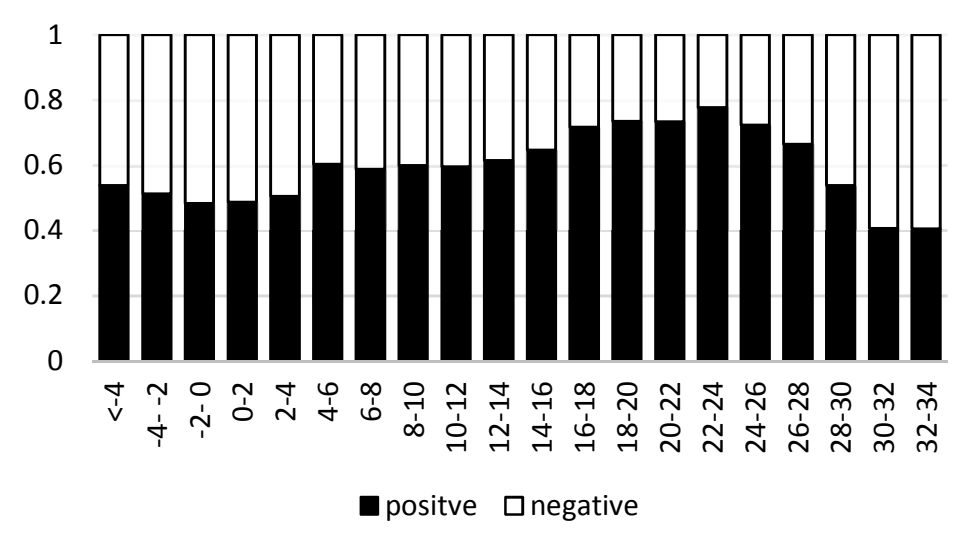

Figure 25.THI variation from Twitter data

The scale built for $\mathrm{THI}$, which is normally used to evaluate comfort in urban areas, has its comfort range between $15^{\circ} \mathrm{C}$ and $20^{\circ} \mathrm{C}$, as Table 5 shows.

Table 5. THI ranges and assessment scale.

\begin{tabular}{|c|c|}
\hline THI & Thermal sensation \\
\hline$>30$ & Torrid \\
\hline $26.4-29.9$ & Very hot \\
\hline $20-26.5$ & Hot \\
\hline $15-20$ & Comfortable \\
\hline $13-15$ & Cool \\
\hline $1.7-13$ & Cold \\
\hline$-10-1.7$ & Very cold \\
\hline$-10--20$ & Extremely cold \\
\hline$-20-40$ & Glacial \\
\hline$<-40$ & Hyper glacial \\
\hline
\end{tabular}

When applying the THI ranges to the Twitter data and comparing them with the obtained results, it becomes apparent that there is a shift to the right (Figure 26), as more people declared to be comfortable or happy about weather when the values of THI ranged between $20^{\circ} \mathrm{C}$ and $26.5^{\circ} \mathrm{C}$.

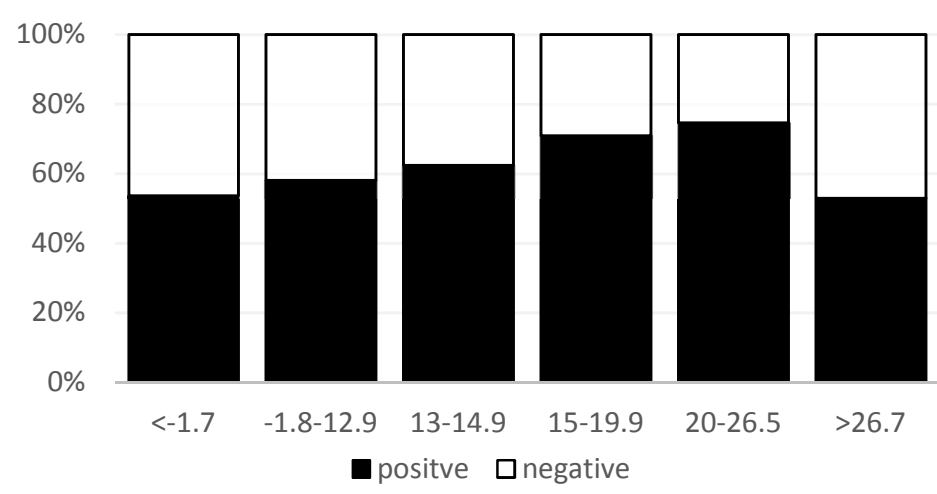

Figure 26. THI ranges applied to the Twitter data

In this case, the shift is not easy to explain, since the discomfort index is rarely used to define comfort ranges in different regions, but to compare urban and rural areas (Unger, 1999) (Toy et al., 2007) (Yilmaz, 2007), in which case the comfort ranges are simply applied to weather data without suffering any modification. 
However, an explanation could be related to the regional wind conditions. It was mentioned before (Chapter 4.2) that the Northeast region is one of the windiest areas in the USA and, therefore, people would need higher temperatures to reach the comfort sensation. This is reflected in the THI ranges because it only includes humidity and temperature in its formula.

\subsection{Weather parameters}

\section{Temperature}

In Figure 27, Sasaki et al. (2000) illustrated the variation of comfort along the temperature scale obtained from their study, as one may see, the percentage of comfortable responses is highest when temperatures are between $20^{\circ} \mathrm{C}$ and $22^{\circ} \mathrm{C}$. This percentage decreases consistently when $\mathrm{t}<20^{\circ} \mathrm{C}$ and $\mathrm{t}>22^{\circ} \mathrm{C}$. Moreover, they identify $\mathrm{t}=12{ }^{\circ} \mathrm{C}$ as a second peak that marks a drastic increase of the (slightly) uncomfortable responses. They argue that with temperatures below $12{ }^{\circ} \mathrm{C}$ people may be more sensitive to wind.

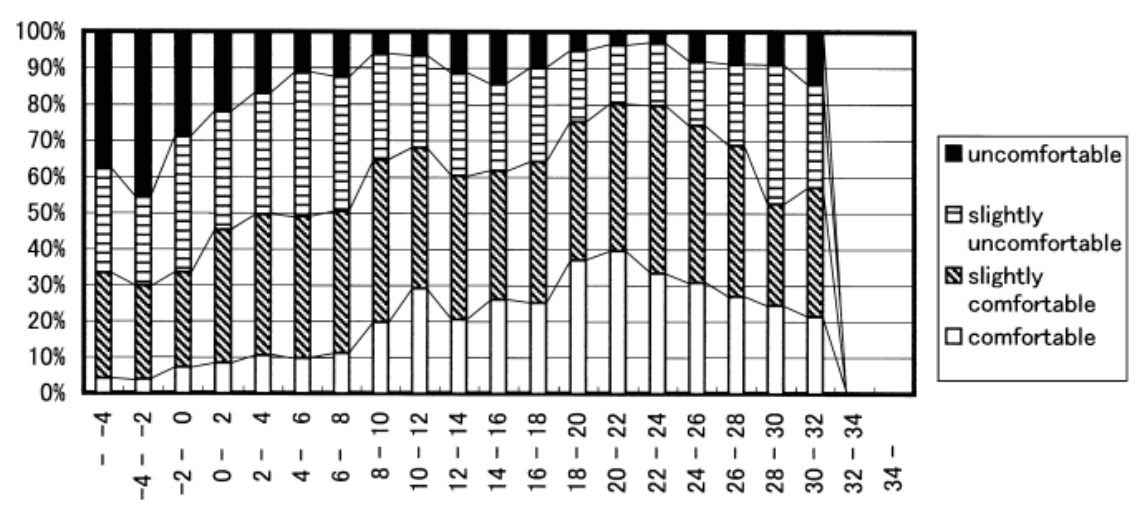

Figure 27. Variation of comfort sensation with air temperature. Source: SASAKI ET AL. (2000)

In figure 28, which shows the results obtained from the Twitter data, the temperature comfort ranges match, since the percentage of positive tweets is highest at temperatures ranging from 20 to $22{ }^{\circ} \mathrm{C}$. Moreover, also the consistent decrease of the comfort sensation when $\mathrm{t}$ is lower than $20{ }^{\circ} \mathrm{C}$ and higher that $22{ }^{\circ} \mathrm{C}$ is illustrated. It is not possible to identify a second peak, as in Sasaki's figure. Nevertheless, in both graphs there is a variation of the decreasing tendency at $-4{ }^{\circ} \mathrm{C}<\mathrm{t}<-2{ }^{\circ} \mathrm{C}$. In Figure 27 , there is a sudden decrease in the comfortable responses, while Figure 28 there is an increase. This can reflect the effect of some other parameter on the thermal perception at that specific temperature range. It requires further exploration.

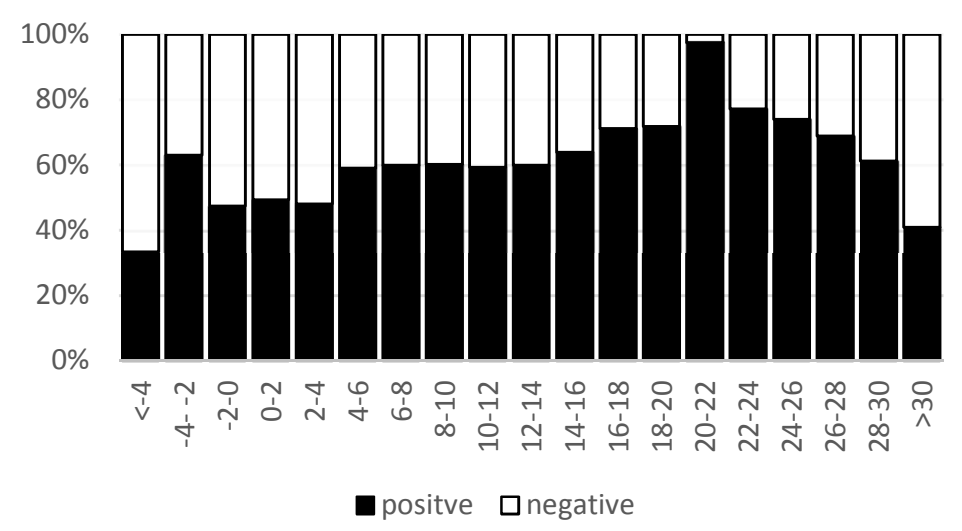

Figure 28. Variation of comfort sensation with Temperature from Twitter data 


\section{Humidity}

In Figure 29, Sasaki et al. (2000) presented the variation of comfort with relative humidity. The percentage of the comfortable response decreases with the increasing of humidity. This decrease is very significant, as it goes from $90 \%$ to $30 \%$ (including the comfortable and slightly comfortable responses). On the other hand, the results obtained from the tweets (figure 30) shows the same general tendency with the decrease of comfort sensation with the increase of relative humidity, but the decrease Is not as steep as in Sasaki's figure (from $82 \%$ to $60 \%$ ).

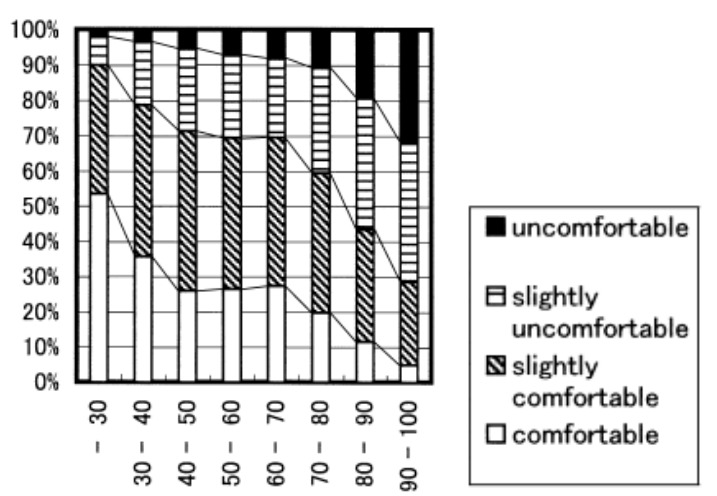

Figure 29. Variation of comfort sensation with Humidity. Source: SASAKI ET AL. (2000)

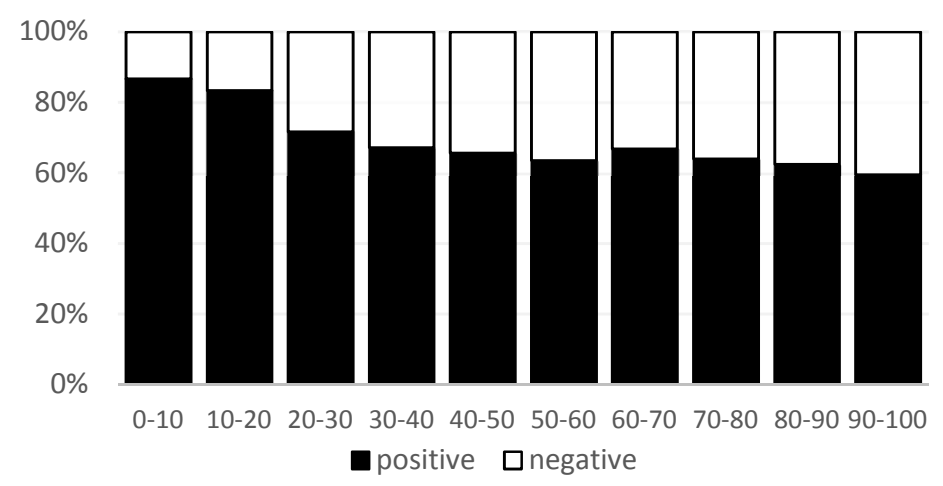

Figure 30. Variation of comfort sensation with Humidity from Twitter data

\section{Wind}

In Figure 31, Sasaki et al. (2000) illustrated the variation of comfort with wind speed, in which the percentage of the comfortable responses slightly decreases with the increasing of wind. Similarly, Figure 32, which illustrates the tweets data, shows the same tendency with a vague decrease of comfort with the increase of wind. 

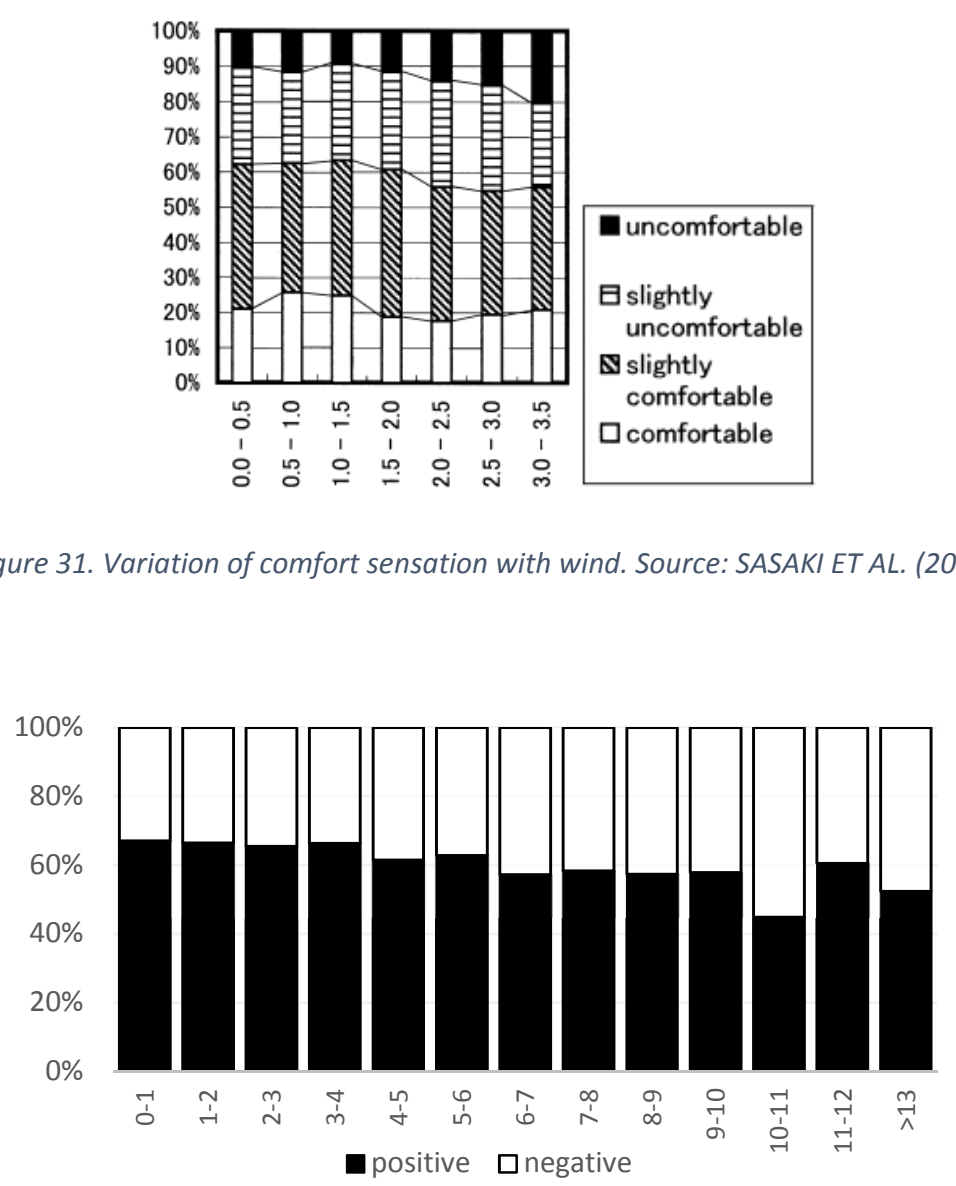

Figure 32. Variation of comfort sensation with wind from Twitter data

It is important to highlight that the impact of wind on comfort sensation is related to temperatures and, therefore, related to the season. Thus, a major variability would be shown in yearly data rather than in seasonal data. Furthermore, this could also be interpreted as the result of people's adaptation to the local windy weather.

\section{Cloud coverage and weather description}

In figure 33, the authors (Sasaki et al., 2000) showed the dependence of comfort on "weather". It is very clear that most of the people interviewed ( $80 \%$ including comfortable and slightly comfortable) felt comfortable during sunny days and uncomfortable during rainy or snowy days.

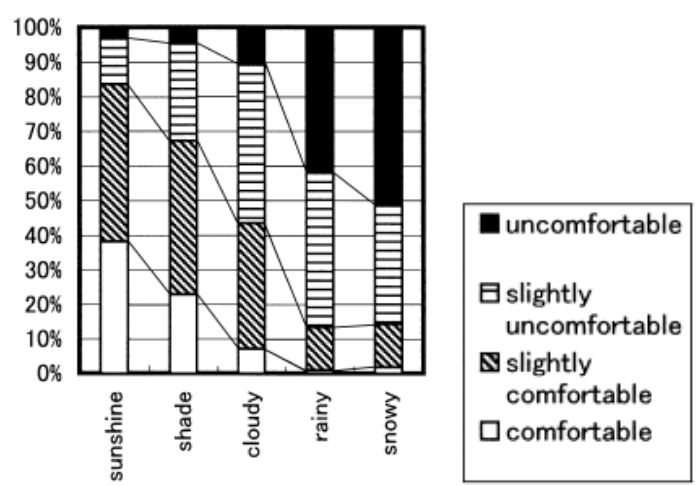

Figure 33. Variation of comfort sensation with weather description. Source: SASAKI ET AL. (2000) 
In Figure 35, the same tendency is shown, even though it is not as clear: $70 \%$ of the tweets were positive during sunny days. Scattered clods or few clouds were considered very comfortable as well. The percentage starts decreasing with broken clouds, light rain and mist (between $60 \%$ and $50 \%$ ) and arrives to $50 \%$ with light snow, overcast sky, moderate rain and heavy rain. Haze and fog seem to be the least comfortable weather conditions (under $50 \%$ ). The weather description chart (figure 34) confirms the tendency, showing that people prefer clear days over overcast days, especially, when the cloud coverage is over $70 \%$.

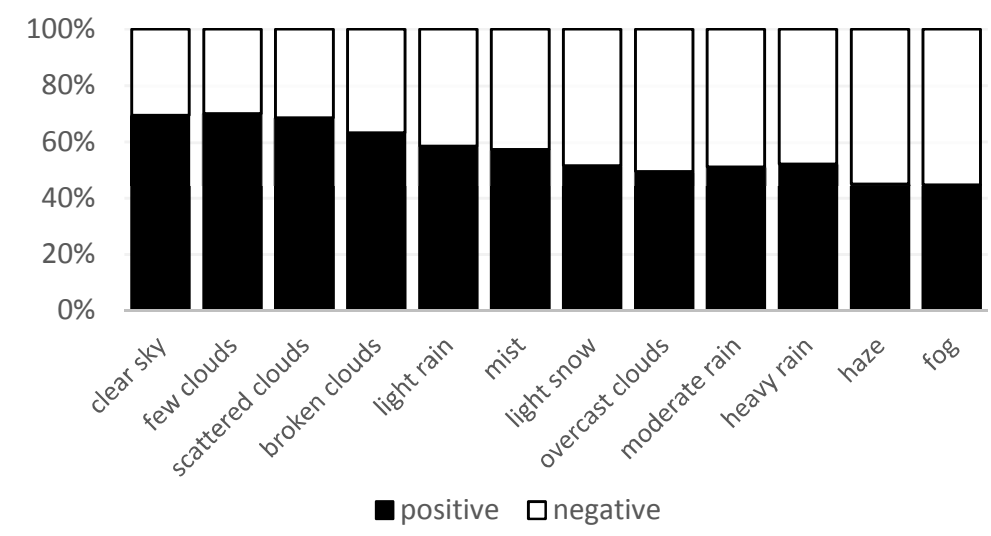

Figure 34. Variation of comfort sensation with weather description from Twitter data

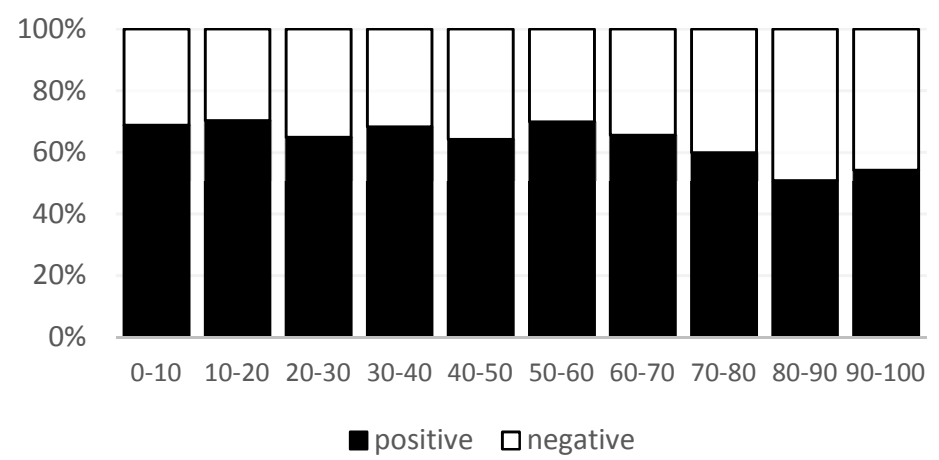

Figure 35. Variation of comfort sensation with cloud coverage from Twitter data

In a nutshell, the obtained comfort ranges for the single weather parameters are quite similar to the ones found in the research of Sasaki et al. (2000). The temperature comfort ranges match perfectly at $20^{\circ} \mathrm{C}-22$ ${ }^{\circ} \mathrm{C}$ and the rest of the features follow the same general tendencies. Wind data illustrates in both cases the same slight decrease of the comfort sensation with the increase of the wind speed. Both humidity charts illustrate that comfort sensation decreases with the increase of humidity. Even if the results of Sasaki et al. (2000) are more pronounced. Finally, tendencies regarding cloud coverage and weather description are again the same as people feel more comfortable or happier on sunny days or when there is a low percentage of cloud coverage and vice versa. Nevertheless, the tweets only show a slight decrease of the comfort with the increase of the cloud coverage, while the decrease in the results of Sasaki et al. (2000) is quite emphasized.

The analysis above was made considering the normalized data to address the different number of positive and negative tweets. In the next paragraphs, the charts considering absolute numbers of tweets will be presented. 
In these graphs (Figure 36), it is possible to see the distribution of the tweets along the different ranges. So, one may argue that people were tweeting the most when temperatures were between $12{ }^{\circ} \mathrm{C}$ and $14{ }^{\circ} \mathrm{C}$, in conditions of high humidity, wind speeds from 2 to $5 \mathrm{~m} / \mathrm{s}$, sunny days and very high or very low cloud coverage. Nevertheless, it may be related to the actual weather conditions. For example, people might have tweeted the most during sunny days, only because autumn 2016 showed an unusual mild weather, which was characterized by a big number of sunny days. In fact, $44 \%$ of tweets, both positive and negative, were posted on sunny days. Similarly, high humidity conditions are common in the humid continental climate. Regarding the cloud coverage, on the other hand, one may think that people would tweet when they feel very comfortable / happy or very uncomfortable / unhappy, which explains why people tweeted the most on cloud coverage extremes.

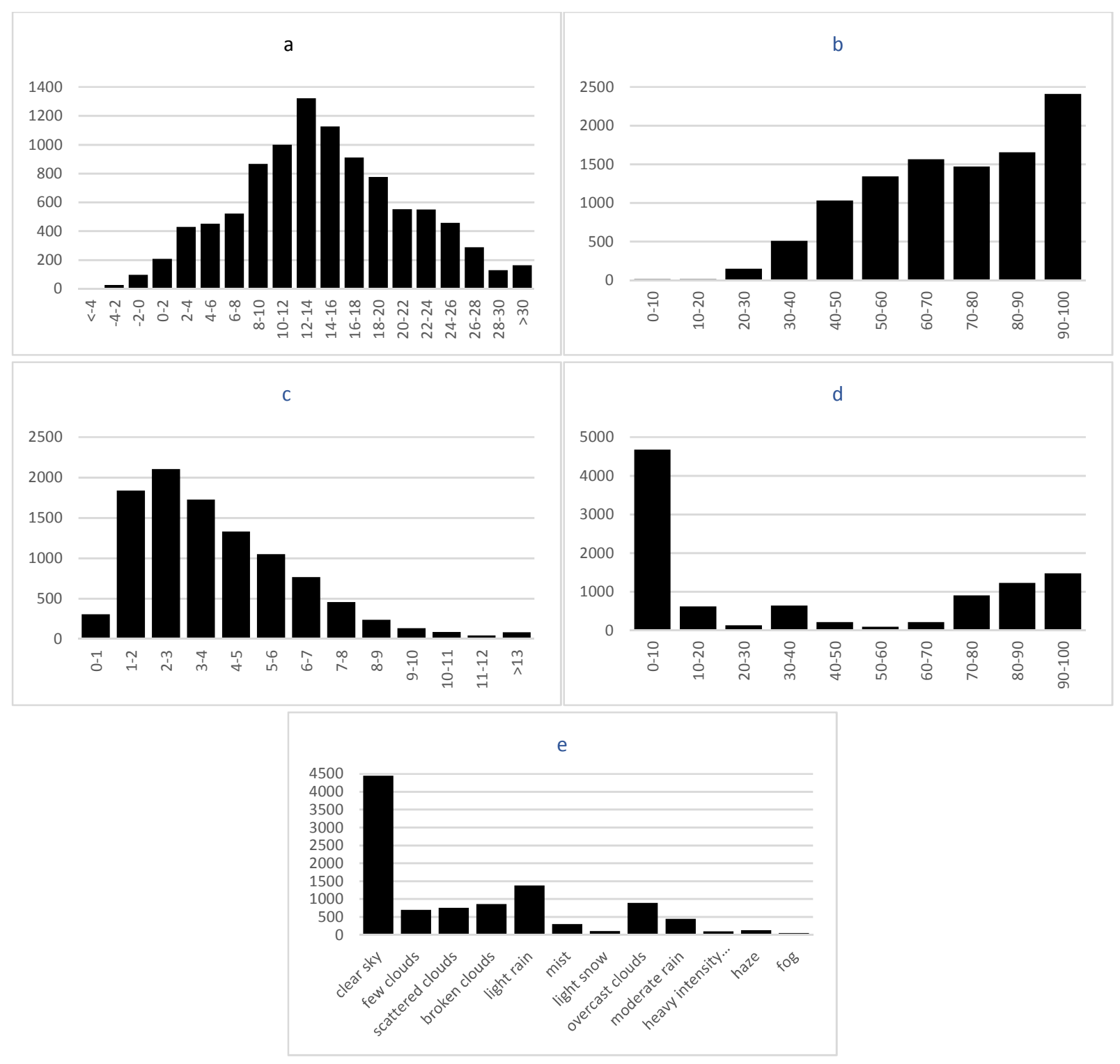

Figure 36. Total number of tweets for all weather parameters (a. Temperature, b. Humidity, c. Wind speed, $d$. Cloud coverage and e. Weather description) 


\subsection{Surveys vs. tweets}

In many ways, tweets are like traditional data collected through surveys, interviews and structured observations, but they also contain unique properties and new features.

Survey methodology implies that a question is asked and answered, thus researchers collect the relevant information for their research. On the other hand, tweets are voluntary information, so they can give answers that nobody has asked for, and since they are spontaneous, they do not answer to any defined research question and, therefore, are unfocused. Moreover, its findings represent only those individuals who offer their opinion.

Surveys can be structured to be representative for an entire population. In contrast, tweets must be carefully handled when it comes to representation issues due to the digital production inequality. Nevertheless, since they are related to big audiences, they can be used to analyze populations that are sometimes underrepresented or hard to reach in surveys, study collective experiences based on a timely event, examine the impact of short-term events on people, and evaluate behaviors of subgroups.

Surveys include few respondents, but have a great control over the provided information. For example, they can gather demographic data and compare the resulting demographic groups. Nevertheless, their small samples may not produce enough variability to entirely study less commonly observed phenomena (McCormick, 2015). Tweets, on the other hand, are completely unsolicited, so they often lack the demographic data that scientists need. Nonetheless, they offer unprecedented volume and variability of data.

Tweets are a rich source of information that offers the opportunity to observe human behavior and human interaction in real time and on a global scale. Surveys, on the other hand, can track opinions and actions over time but are time consuming and costly to administer and cannot provide the same minute-to minute insight that Twitter data can (McCormick, 2015).

With the appropriate infrastructure, one can analyze social media data and begin presenting results within months rather than years, which are typically required for survey-based data collection. Also, while tweets can be captured and stored, face-to-face interactions, once they passed, cannot be reconstructed. Tweets are also updated multiple times per day, which allows the researcher to track opinions and actions as they emerge and develop (Ahmed, 2015).

In a nutshell, twitter data are abundant, relatively easy to access and often free to collect, but are not suitable for all research questions. In the context of this thesis, it is important to understand whether tweets are equally or even more suitable than questionnaires and observations for establishing reference comfort ranges based on human perception. In the first part of the results and discussion chapter, it was shown that the comfort ranges obtained from Tweets are comparable to the ones found in previous studies, but to completely answer this question, a detailed comparison between the strategies used in previous studies and the one used in the present study must be done.

\section{Amount of data, time and space}

The number of questionnaires and interviews applied and completed for this kind of study is unusually high. Thorsson et al. (2007) carried out from 20 to 40 interviews per day, in total they completed almost 4800 interviews. Palutikof et al. (2004) in their study sent questionnaires to 2000 citizens and received 295 back, 
while Sasaki et al. (2000) gave 89 questionnaires and Walton et al. (2007) interviewed 649 people. Nikolopoulou and Lykoudis (2006) created a database containing 10.000 questionnaires and interviews.

Moreover, questionnaires and interviews are limited to specific locations and periods of time. Thorsson et al. (2007) conducted field surveys during the months of March and May in 2 years, between $11 \mathrm{hrs}$. and 15 hrs. on weekdays. Sasaki et al. (2000) asked urban dwellers in three different cities located in Northern Japan to fill a form every day during six months. Stathopoulos et al. (2004) completed 466 interviews during 34 days in Montreal. Most of the records their records were collected during the afternoon. Walton et al. (2007) interviewed people that were seating in two urban parks located in the city of Wellington, between 11 and 15 hrs. Nikolopoulou and Lykoudis (2006) carried out their research in seven different cities, across five European countries throughout the whole year.

In contrast, for this thesis, tweets were collected during three months uninterruptedly and more than 13000 relevant tweets were obtained. These tweets were posted within the study region, covering hundreds of different localities. It is important to highlight that the same methodology could be applied for any requested time, covering all the different parts of the day and night and all the seasons, and any requested area of the world. Nevertheless, as said before, Twitter is not used as much outside of the USA and, therefore, some areas could be poorly covered. Unfortunately, Twitter data may not be applicable for the assessment of microclimates of a specific space, such as a square or a park, because it may be hard to collect enough tweets related to it.

\section{Related information}

Demographic information, clothing, exposure time and surrounding features are related information were collected by the researchers through questionnaires and interviews. Thorson et al. (2007) collected age, clothing, residence state, reason of being at the place, time exposition, aesthetic qualities of the surroundings, thermal perception, weather preferences and emotional state. Sasaki et al. (2000) asked questions related to date, time, perception of each of the environmental conditions and comfort sensation. Nikolopoulou and Lykoudis (2006) collected information about people's perception of thermal environment and comfort conditions, considering the effect of each weather parameter on the overall feeling of satisfaction or dissatisfaction. Walton (2007) collected clothing and exposure time. Mehta (2007) collected design features that influence people's attendance, such as the disposition of the surrounding buildings, sunlight incidence, shade accessibility, wind conditions. He also obtained specific values for exposure time. Palutikof et al. (2004) built a sample considering equal numbers of randomly selected addresses. They obtained demographic information to evaluate possible gender or age bias.

On the other hand, this kind of information was not considered for this thesis because tweets do not specifically provide it. So, the evaluation of subgroups behavior was not possible, as well as the assessment of gender or age bias. Moreover, as it was said before, the collected tweets provide a random sample of all the tweets related to a topic, so the results cannot be generalized, but related to a specific pool, for example, individuals that reported their weather perception on Twitter.

Furthermore, questionnaires and interviews mainly reflected comfort perception related to each weather parameter. Only a reduced number also included weather preferences in compliance with personal activities (Figure 38), emotional state and aesthetics of the surroundings, which are factors that have a great influence on people's perception of outdoor spaces. Tweets on the other hand, are spontaneous, so the opinions they reflect generally include all these factors (Figure 37). Consequently, researchers may correlate the single 
queries and arrive to a rough approximation of the integrated effect of psychological, physiological and physical factors, but these can hardly be individually extracted from tweets.

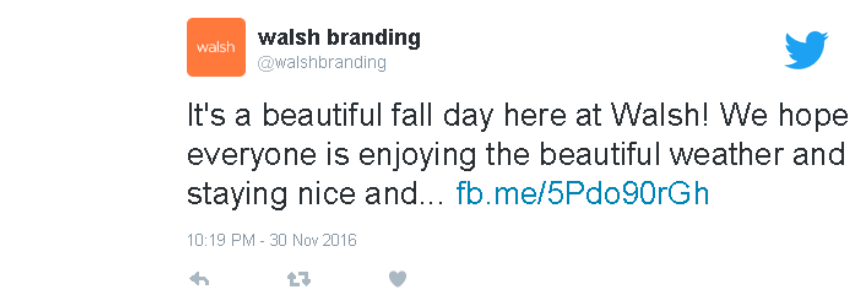

Figure 37. Example of the opinions reflected by tweets. Source: https://twitter.com

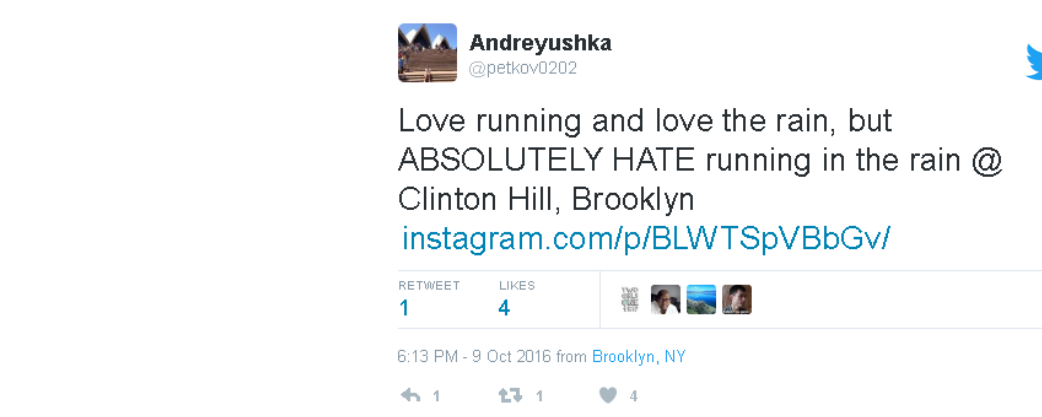

Figure 38. Tweet example of weather preferences in compliance with personal activities. Source: https://twitter.com

\section{Weather parameters and stations}

In the considered previous studies, researchers measured weather parameters by considering near stations or by making on-the-spot measurements with portable instruments. De Montigny et al. (2011) consulted the nearest meteorological stations to obtain the weather data. Nevertheless, temperature data was available only as daily mean, which means that spot or real time measures were not possible. Stathopoulos et al. (2004) decided to focus on periods in which temperatures were moderate to study the influence of humidity, wind seed and solar radiation on the comfort sensation. Walton (2007) measured perceptions of weather parameters on-the-spot, especially wind, sunlight and temperature. Sasaki et al. (2000) considered wind speed and direction, air temperature, relative humidity and weather description. All his respondents lived close to a meteorological station, so the measurements from those were used. Nikolopoulou and Lykoudis (2006) considered the effect of solar radiation, humidity, wind speed and temperatures on the overall feeling of satisfaction or dissatisfaction. They collected detailed microclimatic data with the use of a portable miniweather station.

For this work, data was extracted from Open Weather Map using the geographical coordinates of the tweets. This approach guaranteed real-time values for each weather parameter and for each tweet location due to the great amount of stations included in the OWM network and the addition of the interpolation algorithms. Thus, these measurements are as accurate as most of the previous studies that only considered the nearest station, but less accurate that the on-the-spot measurements. Moreover, even if solar radiation is an important parameter for the evaluation of human comfort, it was not included in the present analysis, as it was mentioned before.

\section{Further elements to consider}

Tweets allow researchers to connect people's perception of weather parameters to the parameters values and unobtrusive observations, which as de Montigny et al. (2011) highlighted, are the most suitable 
conditions to study HCOS. Furthermore, tweets are related to a wide set of different activities performed in a great range of different places. People may tweet about a walk through a park, a tracking excursion in the mountains or a dinner outside in the terrace, while questionnaires can only study specific activities, in specific places, such as the attendance to certain public spaces. Additionally, questionnaires only consider people that have already decided to go out either due to obligation or willingness, but tweets can also give an insight about people that decided to stay at home because weather was awful. 


\section{Conclusion and future work}

From the outcomes of this thesis, it can be concluded that tweets can be used for the assessment of HCOS. An appropriate methodology has been developed and applied in this thesis. However, limitations apply and the assessment works well only under certain conditions.

The limitations of this methodology are related to three different issues: the particular nature of tweets, the meteorological data and the classifier.

Regarding the nature of tweets, even if they contain information about what the authors are doing, the time and place of the activities they describe do not always match the time and place of the post. Consequently, the incidence of these cases must be reduced by identifying specific keywords that can allow the exclusion of this kind of data. Moreover, tweets do not specifically provide demographic information, so if a research requires the analysis of subgroups behavior, this methodology may not be appropriate. Furthermore, when working with Twitter data, it is important to consider that the observations generated through tweets cannot be generalized, but can only be related to a specific group of Twitter users. Finally, tweets may not be suitable for the assessment of microclimates of specific urban places, such as squares, parks or streets, since the results would be limited by the number of available tweets and the weather measurements accuracy.

About the meteorological data, solar radiation, which is fundamental for the evaluation of HCOS, must be collected, either by using only stations measuring it or by the substitution of the meteorological data source. This would allow the application of more complex indices based on heat balance, such as UTCI.

Concerning the classifier, it has to be improved by adding data from all seasons, which would allow the automation of the whole process. The Watson classifier could be directly linked to the tweets collection, providing a real-time assessment of HCOS. This means that for each tweet, a call will be made to the Watson API, which will state whether it is relevant, positive, negative or neutral. Then only positive and negative tweets will be used to make calls by geographical coordinates to the Open Weather Map API and obtain the current meteorological data for the tweets location.

On the other hand, this new methodology offers several advantages over the conventional procedure. First of all, it was shown that tweets give further insights into people's perception of weather by integrating comfort sensation, weather preferences in compliance with personal activities, emotional state and aesthetics of the surroundings. These factors have a great influence on people's perception of outdoor spaces and can be used to improve the current understanding of Human Comfort.

Tweets also allow researchers to connect people's perception of weather to the measured weather parameters in an unobtrusive way. This can reveal behaviors that were non-detected before or shed some light on why not everybody feels comfortable or happy under the same weather conditions. In this regard, tweets can be used to create clusters of weather conditions to connect positive and negative tweets to personal preferences or activities. For example, days characterized by high humidity, medium cloud coverage and fresh temperatures may be preferred by people performing specific physical activities such as jogging or running.

Furthermore, tweets are related to a wide set of different activities performed in a great range of different places and times. This can allow biometeorologists to obtain people's metabolic rate (the amount of energy expended varies with activity level), an important aspect in the assessment of the impact of weather on 
human health. Similarly, biometeorologists can link the geographical location of tweets to air quality conditions data to complete their analysis.

The proposed methodology provides huge amounts of data, translated in better spatial and temporal coverage. Consequently, it could be applied into all the climatic regions of the USA, over a whole year. This would reduce the cultural influences and, therefore, allow the analysis of the effects of both adaptation and seasonal expectations across the country. Similarly, the influence of a heterogeneously composed society on human comfort can be explored, in order to improve the assessment of HCOS in different regions.

Finally, an evaluation of the combined effect of weather parameters could also be done. This may produce new information on which parameters have the biggest effect on the overall comfort perception, which ones have negative or positive impacts on the comfort sensation and eventual interdependencies between specific couples or groups of parameters. This evaluation may give new insights on how parameters are weighted, which would allow the improvement of HCOS assessment.

In a nutshell, Twitter has been proved to be an important data source when it comes to assessing the effect of weather parameters on human outdoor perception. Not only the results of this methodology are comparable to the ones obtained in previous studies, but also the procedure itself shows new features and unexpected applications. 


\section{Acknowledgments}

This work would not exist without a group of very special people that I would like to thank in the following paragraphs.

First of all, I would like to express my gratitude to Prof. Otto Klemm, because he not only believed in me and gave me an opportunity to develop my thesis, but also guided me and supported me through every step of it. Moreover, he gave me confidence and motivated me to give my best. I appreciate every second he dedicated to me during these 6 months.

Secondly, I want to thank Prof. Edzer Pebesma and Prof. Joaquin Torres-Sospedra for supervising my thesis and helping me.

I would also like to acknowledge the importance of the Twitter API, the Open Weather Map API, the Watson: Natural Language Classifier API and the Crowdflower platform for my project. These services provided me with the data and the tools that I needed it to assess HCOS in a new way.

Special thanks go to:

Hanna, because she always had the best advice and shared her knowledge without any conditions. I admire her dedication and appreciate her friendship.

Karsten and Heike, because they always offered help accompanied by a warm smile.

Carl, because his commitment and enthusiasm were contagious.

Jana and Abhash because they were there for me.

Carina, Christina, Marie and Sophie, because they brought me joy in times of worry.

Annette and Klaus, because they opened their home to me and Germany would not have been the same without them.

My parents, because they give me the strength to be better and it was in the hope of helping them that I did this Master.

Last but not least, I want to say thanks to Jan, because he was there every day. He supported me in tough times, gave me great ideas to improve mine, helped me noticing mistakes, stayed awake with me in times of fear, listened to me when I was thinking out loud, bought food in times of hunger and gave me many reasons to keep going and reach my goals. If it had not been for him, I would not have finished this work and, therefore, this master. 


\section{References}

Abu-Asab, Peterson, P. M., Shetler, S. G., \& Orli, S. S. (2010). National climate assessment. Retrieved January 19, 2017, from http://nca2014.globalchange.gov/report/regions/northeast

Adam, N. R., Shafiq, B., \& Staffin, R. (2012). Spatial computing and social media in the context of disaster management. IEEE Intelligent Systems, 27(6), 90-96.

Ahmed, W. (2015, July 10). Using Twitter as a data source: An overview of current social media research tools. Retrieved January 14, 2017, from http://blogs.Ise.ac.uk/impactofsocialsciences/2015/07/10/social-mediaresearch-tools-overview/

Ahmed, W. (2015, September 28). Challenges of using Twitter as a data source: An overview of current resources. Retrieved January 14, 2017, from http://blogs.Ise.ac.uk/impactofsocialsciences/2015/09/28/challenges-of-using-twitter-as-a-data-sourceresources/

Al for your business. (2017). Retrieved January 23, 2017, from https://www.crowdflower.com/

Arens, E., \& Bosselmann, P. (1989). Wind, sun and temperature-Predicting the thermal comfort of people in outdoor spaces. Building and Environment,24(4), 315-320

Association, A. S. (2012). Social science with social media. Retrieved January 14, 2017, from http://www.asanet.org/sites/default/files/savvy/footnotes/jan12/socialmedia_0112.html

Average wind speeds - map viewer. Retrieved January 19, 2017, from https://www.climate.gov/mapsdata/dataset/average-wind-speeds-map-viewer

AYLIEN. (2017). We bring intelligence to the web. Retrieved January 22, 2017, from http://aylien.com/).

Baranowska, M., \& Gabryl, B. (1981). Biometeorological norm as tolerance interval of man to weather stimuli. International journal of biometeorology, 25(2), 123-126.

Bekafigo, M. A., \& McBride, A. (2013). Who tweets about politics? Political participation of Twitter users during the 2011gubernatorial elections. Social Science Computer Review, 0894439313490405.

Belda, M., Holtanová, E., Halenka, T., \& Kalvová, J. (2014). Climate classification revisited: from Köppen to Trewartha. Climate research, 59(1), 1-13.

Biever, C. (2010). Twitter mood maps reveal emotional states of America. New Scientist, 207(2771), 14.

Blazejczyk, K., Epstein, Y., Jendritzky, G., Staiger, H., \& Tinz, B. (2012). Comparison of UTCI to selected thermal indices. International journal of biometeorology, 56(3), 515-535

Bollen, J., Mao, H., \& Pepe, A. (2011). Modeling public mood and emotion: Twitter sentiment and socioeconomic phenomena. ICWSM, 11, 450-453.

By Ali Zifan (Enhanced, modified, and vectorized). (Derived from World Koppen Classification.svg.) [CC BY-SA 4.0 (http://creativecommons.org/licenses/by-sa/4.0)], via Wikimedia Commons

Center, N. R. C. (2015). NRCC home page. Retrieved January 19, 2017, from http://www.nrcc.cornell.edu/

Center, N. R. C. (2015). NRCC narrative overview. Retrieved January 19, 2017, from http://www.nrcc.cornell.edu/regional/narrative/narrative.html

Cox, J., \& Plale, B. (2011). Improving Automatic Weather Observations with the Public Twitter Stream. IU School of Informatics and Computing.

Crowley, D. N., Dabrowski, M., \& Breslin, J. G. (2013). Decision support using linked, social, and sensor data.

de Montigny, L., Ling, R., \& Zacharias, J. (2011). The effects of weather on walking rates in nine cities. Environment and Behavior, 0013916511409033 
Diener, E., \& Suh, E. (1997). Measuring quality of life: Economic, social, and subjective indicators. Social indicators research, 40(1-2), 189-216.

Document. Natural language Classifier | IBM Watson developer cloud. Retrieved January 21, 2017, from https://www.ibm.com/watson/developercloud/doc/natural-language-classifier/index.html

Enloe, S.-L. (1984). National centers for environmental information (NCEI). Retrieved January 19, 2017, from https://www.ncdc.noaa.gov/monitoring-references/maps/us-climate-regions.php

Fänger, P. O. (1972). Thermal comfort: analysis and applications in environmental engineering. Mc. Graww Hill, New York.

Feldman, R. (2013). Techniques and applications for sentiment analysis. Communications

Gagge, A. P., Stolwijk, J. A. J., \& Hardy, J. D. (1967). Comfort and thermal sensations and associated physiological responses at various ambient temperatures. Environmental research, 1-20.

Gagge, A. P., Stolwijk, J. A. J., \& Saltin, B. (1969). Comfort and thermal sensations and associated physiological responses during exercise at various ambient temperatures. Environmental Research, 2(3), 209-229.

Gerbaudo, P. (2012). Tweets and the streets: Social media and contemporary activism. Pluto Press.

Go, A., Bhayani, R., \& Huang, L. (2009). Twitter sentiment classification using distant supervision. CS224N Project Report, Stanford, 1(12)

Greenwood, S., Perrin, A., \& Duggan, M. (2016, November 11). Social media update 2016. Retrieved January 16, 2017, from http://www.pewinternet.org/2016/11/11/social-media-update-2016/

Hannak, A., Anderson, E., Barrett, L. F., Lehmann, S., Mislove, A., \& Riedewald, M. (2012, June). Tweetin'in the Rain: Exploring Societal-Scale Effects of Weather on Mood. In ICWSM.

Jendritzky, G., Sönning, W., \& Swantes, H. J. (1979). Ein objektives Bewertungsverfahren zur Beschreibung des thermischen Milieus in der Stadt-und Landschaftsplanung (" Klima-Michel-Modell"). Schroedel. Mayer, H., \& Höppe, P. (1987). Thermal comfort of man in different urban environments. Theoretical and applied climatology, 38(1), 43-49.

Kallas, P. (2016, December 23). Top 15 most popular social networking sites (and 10 Apps!). Retrieved January 17, 2017, from https://www.dreamgrow.com/top-15-most-popular-social-networking-sites/

Klimadiagramme weltweit - Europa. Retrieved January 19, 2017, from http://www.klimadiagramme.de/

Kouloumpis, E., Wilson, T., \& Moore, J. D. (2011). Twitter sentiment analysis: The good the bad and the omg!. Icwsm, 11(538-541), 164.

Krieck, M., Dreesman, J., Otrusina, L., \& Denecke, K. (2011). A new age of public health: Identifying disease outbreaks by analyzing tweets. In Proceedings of Health Web-Science Workshop, ACM Web Science Conference.

Kumari, P., Singh, S., More, D., Talpade, D., \& Pathak, M. (2015). Sentiment Analysis of Tweets. International Journal of Science Technology \& Engineering, 1.

Lachlan, K. A., Spence, P. R., Lin, X., Najarian, K. M., \& Greco, M. D. (2014). Twitter use during a weather event: comparing content associated with localized and nonlocalized hashtags. Communication Studies, 65(5), 519-534.

Marks, P. (2013). Happy, snappy tweets gain the most followers. New Scientist, 217(2906), 24.

Mayer, H. (1993). Urban biometeorology. Experientia, 49(11), 957-963 
McCormick, T. H., Lee, H., Cesare, N., Shojaie, A., \& Spiro, E. S. (2015). Using Twitter for Demographic and Social Science Research Tools for Data Collection and Processing. Sociological Methods \& Research, 0049124115605339

Mehta, V. (2007). Lively streets determining environmental characteristics to support social behavior. Journal of planning education and research, 27(2), 165-187

Nakov, P., Rosenthal, S., Kiritchenko, S., Mohammad, S. M., Kozareva, Z., Ritter, A., ... \& Zhu, X. (2016). Developing a successful SemEval task in sentiment analysis of Twitter and other social media texts. Language Resources and Evaluation, 50(1), 35-65.

Nass, O. Universal thermal climate index. Retrieved January 7, 2017, from http://www.utci.org/index.php

Nevins, R. G., Rohles, F. H., Springer, W., \& Feyerherm, A. M. (1966). A temperature-humidity chart for thermal comfort of seated persons. ASHRAE transactions, 72(1), 283-291

Nikolopoulou, M., \& Lykoudis, S. (2006). Thermal comfort in outdoor urban spaces: analysis across different European countries. Building and Environment, 41(11), 1455-1470.

Nikolopoulou, M., \& Steemers, K. (2003). Thermal comfort and psychological adaptation as a guide for designing urban spaces. Energy and Buildings, 35(1), 95-101.

Northeastern United States. Retrieved January 19, 2017, from http://www.newworldencyclopedia.org/entry/Northeastern_United_States

Ogunsote, O. O., \& Prucnal-Ogunsote, B. (2002). Comfort limits for the effective temperature index in the tropics: A Nigerian case study. Architectural Science Review, 45(2), 125-132.

Pak, A., \& Paroubek, P. (2010, May). Twitter as a Corpus for Sentiment Analysis and Opinion Mining. In LREc (Vol. 10, No. 2010).

Palutikof, J. P., Agnew, M. D., \& Hoar, M. R. (2004). Public perceptions of unusually warm weather in the UK: impacts, responses and adaptations. Climate Research, 26(1), 43-59

Panasyuk, A., Blasch, E., Kase, S. E., \& Bowman, L. (2013, October). Extraction of Semantic Activities from Twitter Data. In STIDS (pp. 79-86).

Paul, M. J., \& Dredze, M. (2011). You are what you Tweet: Analyzing Twitter for public health. ICWSM, 20, 265-272.

Penwarden, A. D. (1973). Acceptable wind speeds in towns. Building Science, 8(3), 259-267

Princeton. (2017). About WordNet - WordNet - about WordNet. Retrieved January 22, 2017, from https://wordnet.princeton.edu/

Raspberry pi foundation - about us. (2012). Retrieved January 16, 2017, from https://www.raspberrypi.org/about/

Resch, B., Summa, A., Sagl, G., Zeile, P., \& Exner, J. P. (2015). Urban emotions-Geo-semantic emotion extraction from technical sensors, human sensors and crowdsourced data. In Progress in location-based services 2014 (pp. 199-212))

Roberts, K., Roach, M. A., Johnson, J., Guthrie, J., \& Harabagiu, S. M. (2012, May). EmpaTweet: Annotating and Detecting Emotions on Twitter. In LREC (pp. 3806-3813).

Sasaki, R., Yamada, M., Uematsu, Y., \& Saeki, H. (2000). Comfort environment assessment based on bodily sensation in open air: relationship between comfort sensation and meteorological factors. Journal of Wind Engineering and Industrial Aerodynamics, 87(1), 93-110 
Skoric, M., Poor, N., Achananuparp, P., Lim, E. P., \& Jiang, J. (2012, January). Tweets and votes: A study of the 2011 singapore general election. In System Science (HICSS), 2012 45th Hawaii International Conference on (pp. 2583-2591). IEEE.

Stathopoulos, T., Wu, H., \& Zacharias, J. (2004). Outdoor human comfort in an urban climate. Building and Environment, 39(3), 297-305

Streaming APIs - Twitter developers. (2016). Retrieved January 16, 2017, from Twitter, https://dev.twitter.com/streaming/overview

Thorsson, S., Honjo, T., Lindberg, F., Eliasson, I., \& Lim, E. M. (2007). Thermal comfort and outdoor activity in Japanese urban public places. Environment and Behavior

Toy, S., Yilmaz, S., \& Yilmaz, H. (2007). Determination of bioclimatic comfort in three different land uses in the city of Erzurum, Turkey. Building and Environment, 42(3), 1315-1318.

Tumasjan, A., Sprenger, T. O., Sandner, P. G., \& Welpe, I. M. (2010). Predicting Elections with Twitter: What 140 Characters Reveal about Political Sentiment. ICWSM, 10, 178-185.

Unger, J. (1999). Comparisons of urban and rural bioclimatological conditions in the case of a CentralEuropean city. International Journal of Biometeorology, 43(3), 139-144.

Unit, E. I. (2005). The Economist Intelligence Unit's quality-of-life index.Retrieved July, 17(2005), 245-277.

Villatoro, D., \& Nin, J. (2013). Citizens sensor networks. In Citizen in Sensor Networks (pp. 1-5). Springer Berlin Heidelberg.

Walton, D., Dravitzki, V., \& Donn, M. (2007). The relative influence of wind, sunlight and temperature on user comfort in urban outdoor spaces. Building and environment, 42(9), 3166-3175

Who Tweets? Making Twitter data "useful" for social science. (2016, April 23). Retrieved January 14, 2017, from http://nsmnss.blogspot.de/2016/04/who-tweets-making-twitter-data-useful.html

Winslow, C. E., Herrington, L. P., \& Gagge, A. P. (1937). Physiological reactions of the human body to varying environmental temperatures. American Journal of Physiology--Legacy Content, 120(1), 1-22.

Yilmaz, S., Toy, S., \& Yilmaz, H. (2007). Human thermal comfort over three different land surfaces during summer in the city of Erzurum, Turkey. Atmósfera, 20(3), 289-297.

Current weather and forecast- OpenWeatherMap. (2012). Retrieved January 16, 2017, from https://openweathermap.org/ 